\title{
Exploration of Anti-inflammatory Mechanism of Forsythiaside A and Forsythiaside B in CuSO4- Induced Inflammation in Zebrafish by Metabolomic and Proteomic Analyses
}

\section{Lihong Gong}

Chengdu University of Traditional Chinese Medicine

\section{Linyuan Yu}

Chengdu University of Traditional Chinese Medicine

Xiaohong Gong

Chengdu University of Traditional Chinese Medicine

\section{Cheng Wang}

Chengdu University of Traditional Chinese Medicine

\section{Naihua Hu}

Chengdu University of Traditional Chinese Medicine

\section{Xuyang Dai}

Chengdu University of Traditional Chinese Medicine

Yunxia Li ( $\sim$ lyxcdutcm@126.com )

Chengdu University of Traditional Chinese Medicine https://orcid.org/0000-0003-1307-4335

\section{Research}

Keywords: Forsythiaside A, Forsythiaside B, Zebrafish, Inflammation, Metabolomics, Proteomics

Posted Date: April 22nd, 2020

DOI: https://doi.org/10.21203/rs.2.19758/v2

License: (c) (i) This work is licensed under a Creative Commons Attribution 4.0 International License. Read Full License

Version of Record: A version of this preprint was published at Journal of Neuroinflammation on June 3rd, 2020. See the published version at https://doi.org/10.1186/s12974-020-01855-9. 


\section{Abstract}

Background: Inflammation is a general pathological phenomenon during severe disturbance of homeostasis. Forsythiaside $A(F A)$ and Forsythiaside $B(F B)$ are phenylethanoid components isolated from dried fruits of Forsythia suspensa (Thunb.) Vahl., which show a significant effect against inflammation. However, the antiinflammatory properties and therapeutic mechanisms of FA and FB have not yet been systematically clarified.

Methods: In this study, the anti-inflammatory effects of FA and FB were investigated in CuSO4-induced inflammation in zebrafish. Intracellular ROS and NO generation in zebrafish were investigated using fluorescent probes. Metabolomics and proteomics analyses using liquid chromatography mass spectrometry were carried out to identify the expressions of metabolites and proteins associated with the treatment of FA and FB in CuSO4-induced inflammation in zebrafish larvae. Quantitative polymerase chain reaction (PCR) was performed to detect the progressive changes in genes.

Results: FA and FB inhibited neutrophils migration to the damaged neuromasts and remarkably reduced CuSO4-induced ROS and NO generation in zebrafish. Metabolomic analysis indicated that Nicotinate and nicotinamide metabolism, Energy metabolism, Pyrimidine metabolism and Purine metabolism were mainly involved. Proteomic analysis identified 146 differentially expressed proteins between control and model group. The changed expressions of Collagen (collagen type II alpha 1b precursor (col2a1b), collagen alpha2(IX) chain precursor (col9a2), collagen type IX alpha I precursor (col9a1b)), nucleoside diphosphate kinase 3 isoform X1 (Nme3), WD repeat-containing protein 3 (Wdr3), 28S ribosomal protein S7 mitochondrial precursor (Mrps7) in model group were all reversed after being treated by FA or FB. FA and FB could reverse the abnormal expressions of potential metabolite and protein biomarkers to alleviate CuSO4 damage to the neuromasts in the lateral line of zebrafish.

Conclusions: Our results indicated that FA and FB possessed remarkable anti-inflammatory activities against CuSO4-induced neuromasts damage in zebrafish larvae, and suggested a multi-component and multiregulatory therapeutic mechanism of FA and FB.

\section{Background}

Inflammatory response is a key component in normal homeostasis that protects the body from irritation and restores damaged tissue structure and function. Generally, inflammatory reactions are beneficial to the body. However, excessive and uncontrolled inflammation can cause chronic diseases such as cancer and neurodegenerative diseases, including Parkinson's disease and Alzheimer's disease [1, 2]. During this process, the over-activation of macrophages and neutrophils can induce the secretion of Interleukin-6 (IL-6), Tumor necrosis factor- $\alpha$ (TNF- $\alpha$ ) and Interleukin-1 $\beta$ (IL-1 $\beta$ ), which are significant mediators participating in inflammation [3].

Forsythiae Fructus is the dried fruit of Forsythia suspensa (Thunb.) Vahl. As a heat-clearing and detoxifying traditional Chinese medicine that has the effect of antipyretic and anti-inflammation, Forsythiae Fructus has been used for treating various infectious diseases, such as acute nephritis and ulcers [4]. Forsythiaside A (FA, Fig. 1A) and Forsythiaside B (FB, Fig. 1B) are the major bioactive components extracted from Forsythiae 
Fructus and have been reported to possess anti-inflammatory and anti-bacterial activities $[5,6]$. However, few studies have been conducted to systematically clarify the anti-inflammatory activities and therapeutic mechanisms of FA and FB.

Zebrafish (Danio rerio), with similar morphological and physiological functions to humans, have been widely used for having unique advantages of high fecundity, easy breeding, small size, the availability of various transgenic lines, simple in vivo operation and real-time imaging [7-9]. The obvious transparency of zebrafish embryos and availability of zebrafish transgenic line make it possible to monitor inflammatory processes and observe cells behavior in vivo [10]. Researches have also shown that the zebrafish immune system has significant similarities with humans, and almost all human immune system cells have counterparts in zebrafish [11]. The neuromasts in the lateral line system of zebrafish are composed of mechanosensory hair cells and can be damaged by physical or chemical stimulation [12]. $\mathrm{CuSO}_{4}$ is a chemical metal that induces neuromasts damage in zebrafish accompanied with an invasion of inflammatory cells, which in turn result in a progressive disruption of neuromasts structure [13]. Unfortunately, the specific role of $\mathrm{FA}$ and $\mathrm{FB}^{\text {in } \mathrm{CuSO}_{4}{ }^{-}}$ induced inflammation in zebrafish and their molecular mechanisms still remain to be further explored.

However, the biological interpretation of common pharmacological research may be a great challenge due to the complicated biochemical regulatory mechanism at multiple levels. An integrated analysis of omics data is promising and can provide an improved comprehension of biological mechanisms via identifying the potential biomarkers and interpreting their intrinsic relations. The combination of metabolomics and proteomics is a powerful tool that frequently applied in complex mechanism exploration. Therefore, we investigated the anti-inflammatory mechanisms of FA and FB via an untargeted metabolic and proteomic profiling of zebrafish based on liquid chromatography mass spectrometry. Meanwhile, we proposed a potential mechanism to explain the inflammatory neuromasts behavior in zebrafish during $\mathrm{CuSO}_{4}$ exposure and systematically clarify the therapeutic effects and mechanisms of FA and FB for inflammation, thus providing a potential understanding of FA and FB in the treatment of inflammation and their clinical applications.

Fig. 1 Chemical structures of Forsythiaside $A(A)$ and Forsythiaside $B(B)$.

\section{Methods}

\subsection{Materials}

FA (MUST-18010303) and FB (MUST-18081202) were purchased from Chengdu MUST Bio-technology Co., Ltd (Chengdu, China). Tricaine was obtained from Sigma (Shanghai, China). Acetonitrile, Methanol and Formic acid of HPLC grade were collected from Merck Chemicals (Shanghai, China), Wokai Chemical Technology Co., Ltd. (Shanghai, China) and TCI Chemical Industry Development Co., Ltd. (Shanghai, China), respectively. DAF-FMDA and DCF-DA were purchased from Yeasen (Shanghai, China). Trizol reagents were purchased from Ambion Life Technologies (Carlsbad, CA, USA). 5 × All-In-one MasterMix and Eva Green $2 \times$ qRT-PCR MasterMix-Low RoX were purchased from Applied Biological Materials Inc (Richomnd, BC, Canada). PCR primer sequences were synthesized in TSINGKE Biological Technology (Chengdu, China). Other chemicals and reagents were obtained from Kelong Chemical Reagent Factory (Chengdu, China). 


\subsection{Zebrafish}

Zebrafish larvae of wild-type AB strain and neutrophil-specific transgenic (mpx:EGFP) with neutrophils labelled with enhanced green fluorescent protein were purchased from China Zebrafish Resource Center (Wuhan, China) and raised at $28.5 \pm 1.0^{\circ} \mathrm{C}$ on a $14 \mathrm{~h} \mathrm{light} / 10 \mathrm{~h}$ dark cycle. Zebrafish embryos were collected by natural spawning and maintained in embryonic medium $\left(5.00 \mathrm{mmol} \cdot \mathrm{L}^{-1} \mathrm{NaCl}, 0.44 \mathrm{mmol} \cdot \mathrm{L}^{-1} \mathrm{CaCl}_{2}, 0.33\right.$ $\mathrm{mmol} \cdot \mathrm{L}^{-1} \mathrm{MgSO}_{4}, 0.17 \mathrm{mmol} \cdot \mathrm{L}^{-1} \mathrm{KCl}$ and $0.1 \%$ methylene blue, equilibrated to $\mathrm{pH} 7.0$ ) till drug administration $[14,15]$.

\subsection{Anti-inflammatory effects of FA and $\mathrm{FB}$ in $\mathrm{CuSO}_{4}$-induced inflammation in zebrafish}

\subsubsection{In vivo neutrophil recruitment assay}

All experiments were performed on 3 days postfertilization (dpf) zebrafish larvae in the present study. Transgenic (mpx:EGFP) zebrafish were randomly transferred to the twelve-well plate (15 larvae per well) and assigned to 8 groups for neutrophil recruitment investigation. In control group, larvae were incubated in zebrafish embryonic medium; In model group, larvae were exposed to $10 \mu \mathrm{mol}^{\circ} \mathrm{L}^{-1} \mathrm{CuSO}_{4}$ for $40 \mathrm{~min}$; In FA group, larvae were exposed to $1 \mathrm{~h}$ FA pre-treatment at $120,60,30 \mu \mathrm{mol} \cdot \mathrm{L}^{-1}$ followed by a mixture incubation of $10 \mu \mathrm{mol} \cdot \mathrm{L}^{-1} \mathrm{CuSO}_{4}$ and FA $\left(120,60,30 \mu \mathrm{mol} \cdot \mathrm{L}^{-1}\right)$ for $40 \mathrm{~min}$; In FB group, larvae were exposed to $1 \mathrm{~h} \mathrm{FB}$ pre-treatment at $150,75,37.5 \mu \mathrm{mol} \cdot \mathrm{L}^{-1}$ followed by a mixture incubation of $10 \mu \mathrm{mol} \cdot \mathrm{L}^{-1} \mathrm{CuSO}_{4}$ and $\mathrm{FB}(150$, $75,37.5 \mu \mathrm{mol} \cdot \mathrm{L}^{-1}$ ) for $40 \mathrm{~min}$. All drugs were diluted in embryonic medium. After the treatments, zebrafish larvae were anesthetized with tricaine solution. Then zebrafish were observed under Leica M165Fic fluorescence microscope (Leica Microsystems, Germany) and photographed. The recruitment number of neutrophils to neuromasts in zebrafish was counted by Image Pro Plus 6.0 software (Media Cybernetics, USA).

\subsubsection{Intracellular production of ROS and NO in zebrafish}

Fluorescent probes of 2',7'-dichlorodihydrofluorescein diacetate (DCF-DA) and diaminofluorophore 4-amino-5methylamino-2',7'-difluorofluorescein diacetate (DAF-FMDA) were used to investigate the intracellular reactive oxygen species (ROS) and nitric oxide (NO) accumulation in $\mathrm{CuSO}_{4}$-induced inflammation in zebrafish, respectively [16]. Wild-type AB strain zebrafish were randomly transferred to the twelve-well plate (15 larvae per well) and assigned to 8 groups according to the previous description for modeling and drug administration. After the treatments, zebrafish were moved to the twelve-well plate ( 15 larvae per well) and treated with DCF-DA $\left(0.05 \mu \mathrm{mol} \cdot \mathrm{L}^{-1}\right)$ and DAF-FMDA $\left(5 \mu \mathrm{mol} \cdot \mathrm{L}^{-1}\right)$ solution, respectively. Zebrafish were washed with fresh medium after $1 \mathrm{~h}$ incubation in darkness and subsequently anesthetized with tricaine. The accumulation of ROS and NO in zebrafish were photographed under a Leica M165Fic fluorescence microscope. Finally, Image Pro Plus 6.0 software was used to quantitatively analyze the fluorescence intensity of individual zebrafish larvae.

\subsubsection{Statistical analysis of neutrophils migration and ROS, NO accumulation}


Zebrafish larvae were assigned to each treatment group randomly and pharmacodynamic experiments were repeated three times. Statistical analyses were conducted by SPSS 25.0 (SPSS Inc., Chicago, IL, USA) and graphs were generated by GraphPad Prism 6.0 (GraphPad, San Diego, CA, USA). All statistics regarding neutrophils migration, ROS and NO accumulation were evaluated by independent sample $t$ test or MannWhitney U test (two groups) and one-way ANOVA or Kruskal-Wallis test followed by pairwise comparisons (three or more groups) depending on whether the data were normally distributed. Data were shown as mean \pm S.D and $p<0.05$ were assumed for statistical significance indication.

\subsection{Metabolomic analysis}

\subsubsection{Sample processing and metabolites detection}

Zebrafish larvae from control, model, FA $\left(120 \mu \mathrm{mol} \cdot \mathrm{L}^{-1}\right)$ and FB $\left(150 \mu \mathrm{mol} \cdot \mathrm{L}^{-1}\right)$ group were transferred to 2 $\mathrm{mL}$ centrifuge tubes, respectively. $1 \mathrm{~mL} 80 \%$ methanol and steel balls were added to the tube. Samples were grinded for $1 \mathrm{~min}$ in a Tissue Grinding Device (SCIENTZ-48, Xinzhi Biotechnology Co., Ltd) at $70 \mathrm{~Hz}$. Samples were then placed in the Ultrasonic Machine (KW-100TDV, Shumei Ultrasonic Instrument Co., Ltd) at room temperature for $30 \mathrm{~min}$ and subsequently incubated on ice for $30 \mathrm{~min}$. After being centrifuged for $10 \mathrm{~min}$ $\left(14,000 \mathrm{rpm}, 4^{\circ} \mathrm{C}\right)$, the supernatant was transferred to a new tube. Samples were blow-dried by vacuum concentration and dissolved by $400 \mu \mathrm{L} 2$-chlorobenzalanine methanol aqueous solution $\left(1: 1,4{ }^{\circ} \mathrm{C}\right)$. Then, samples were prepared for Liquid Chromatography-Mass Spectrometry (LC-MS) detection following filtration through $0.22 \mu \mathrm{m}$ microfilter membrane. Quality control samples were obtained by mixing $20 \mu \mathrm{L}$ of each prepared sample.

Chromatographic separation was performed on High-Performance Liquid Chromatography (HPLC, Ultimate 3000 system, Thermo), equipped with a Waters column $(1.8 \mu \mathrm{m}, 150 \times 2.1 \mathrm{~mm})$ of $40^{\circ} \mathrm{C}$. The autosampler temperature was set at $8{ }^{\circ} \mathrm{C}$. Gradient elution of analytes was carried out with $5 \mathrm{mmol} \cdot \mathrm{L}^{-1}$ ammonium formate in water $(A)$ and acetonitrile $(B)$ in negative model or $0.1 \%$ formic acid in water $(C)$ and $0.1 \%$ formic acid in acetonitrile (D) in positive model at a flow rate of $0.25 \mathrm{~mL} \cdot \mathrm{min}^{-1}$. Each sample of $2 \mu \mathrm{L}$ was injected after equilibration. A linear gradient of solvent $B$ (negative model) or solvent $D$ (positive model) was programmed as follows: $0-1 \mathrm{~min}, 2 \%$ acetonitrile or $0.1 \%$ formic acid in acetonitrile (B/D); $1-9 \mathrm{~min}, 2 \%-50 \%$ B/D; 9-12 min, 50\%-98\% B/D; 12-13.5 min, 98\% B/D; 13.5-14 min, 98\%-2\% B/D; 14-20 min, 2\% D-positive model (14-17 min, 2\% B-negative model).

The electrospray ionization mass detection (ESI-MSn) in positive and negative ion mode were performed on Mass spectrometer (Q Exactive Focus, Thermo) with spray voltage of $3.8 \mathrm{kV}$ and $-2.5 \mathrm{kV}$, respectively. Sheath gas and auxiliary gas were set at 45 and 15 arbitrary units, respectively. The capillary temperature was set at $325^{\circ} \mathrm{C}$. The Orbitrap Analyzer (Orbitrap Fusion Lumos mass spectrometer, CA, USA) performed a full scan with a mass range of $81-1000$ mass to charge ratio $(\mathrm{m} / \mathrm{z})$ and a mass resolution of 70,000 . Mass Spectrometry/Mass Spectrometry (MS/MS) experiments were data-dependently acquired by higher energy collisional dissociation (HCD) scanning. The standard collision energy was $30 \mathrm{eV}$. Dynamic exclusion was implemented to remove some unnecessary information in MS/MS spectrum.

\subsubsection{Data processing and multivariate analyses of metabolites}


Data of Ultra-Performance Liquid Chromatography-Mass Spectrometry/Mass Spectrometry (UPLC-MS/MS) were displayed in mzXML format via Proteowizard software (v3.0.8789). Data pre-processing was performed by XCMS program of $\mathrm{R}$ language (v3.3.2) via autoscaling, mean-centering and scaling to unit variance. Parameters were mainly set as follows: $\mathrm{bw}=2, \mathrm{ppm}=15, \mathrm{mzwid}=0.015$, peak width $=\mathrm{c}(5,30)$, $\mathrm{mzdiff}=$ 0.01 , method = cent wave. The area of metabolite peaks in each sample was normalized by the summation method applied in Metaboanalyst (http://www.metaboanalyst.ca/). Multivariate analyses of metabolites including standard peak areas and retention time were performed using $\mathrm{R}$ language to further locate the specific metabolites in the database. The biomarker formulation was generated and queried of accurate molecular weights with mass errors less than 30 part per million (ppm). The fracture patterns of potential biomarkers were analyzed and identifications of potential biomarkers were performed by Human Metabolome Database (HMDB) (http://www.hmdb.ca) and METLIN (http://metlin.scripps.edu/). Kyoto Encyclopedia of Genes and Genomes Database (KEGG) (http://www.kegg.jp/) was used to plot metabolomic pathway network diagram. Pathway enrichment analysis by Metaboanalyst was performed to screen metabolomic pathway of identified metabolites in the current study.

\subsubsection{Statistical analysis of metabolomics}

For metabolomic analysis, 10 biological replications of each group were carried out in the present study. Orthogonal projections to latent structures discriminant analysis (OPLS-DA) was used to filter metabolites significantly contributed to group discrimination by variable importance in the projection (VIP) values. The SPlot (R language: v3.3.2) was applied to check the differentiating metabolites with the value of VIP $\geq 1$. Oneway ANOVA of SPSS 25.0 was conducted to further determine whether the potential metabolites were significantly different $(p \leq 0.05)$ among control, model, FA and FB group. Finally, metabolites with VIP $\geq 1$ and $p \leq 0.05$ were considered as potential metabolite biomarkers $[17,18]$.

\subsection{Proteomic analysis}

\subsubsection{Protein extraction and digestion}

Proteins were extracted from zebrafish larvae of control, model, FA $\left(120 \mu \mathrm{mol} \cdot \mathrm{L}^{-1}\right)$ and FB $\left(150 \mu \mathrm{mol} \cdot \mathrm{L}^{-1}\right)$ group as previously described [19]. Briefly, $500 \mu \mathrm{L}$ Lysis Buffer (2\% sodium deoxycholate, $50 \mathrm{mM}$ ammonium bicarbonate, $75 \mathrm{mM}$ sodium chloride) was added to samples, which were then ground on ice in Ultrasonic Crushing Machine (Scientz-JY92, Ningbo Xinzhi Biotechnology Co., Ltd) for 10 min with $2 \mathrm{~s}$ on and $4 \mathrm{~s}$ off at $15 \%$ power. The ground material was centrifuged for $10 \mathrm{~min}\left(10,000 \times \mathrm{g}, 4^{\circ} \mathrm{C}\right) .10 \mathrm{mM}$ dithiothreitol was added to the supernatant to precipitate proteins at $-20^{\circ} \mathrm{C}$, and the precipitation procedure was repeated with acetone until the supernatant became colorless as previously described [20].Then the supernatant was incubated at $56^{\circ} \mathrm{C}$ for $1 \mathrm{~h}$ and subsequently alkylated with $55 \mathrm{mmol} \cdot \mathrm{L}^{-1}$ iodoacetamide for $45 \mathrm{~min}$ treatment in darkness at room temperature. Proteins were then resuspended with Lysis Buffer and the concentration of protein was detected using BCA assay. The protein solution $(100 \mu \mathrm{g})$ was digested by Trypsin Gold (40:1, protein:trypsin) at $37^{\circ} \mathrm{C}$ overnight. Peptides desalination was carried out via a Strata $\mathrm{X}_{18}$ column and then vacuum-dried in accordance with manufacturer's instructions.

\subsubsection{TMT labeling and fractionation}


The peptides were labeled using Tandem Mass Tags (TMT) six plex Isobaric Label Reagent Set (Thermo Scientific, 90061) in accordance with manufacturer's instructions. Samples of control and model group were labeled with tags 126 and 127, while samples of FA and FB group were labeled with tags 128 and 129. The labeled peptides were separated using Shimadzu LC-20AB HPLC Pump system (Shimadzu, Kyoto, Japan) equipped with a high-pH reverse phase (RP) column ( $5 \mu \mathrm{m}$, Phenomenex, CA, USA). Then labeled peptides were firstly reconstituted to $2 \mathrm{~mL}$ with buffer $\mathrm{A}$ ( $5 \% \mathrm{ACN}$ and $95 \% \mathrm{H}_{2} \mathrm{O}$, with the pH adjusted to 9.8 with ammonia) and then loaded on the column for separation with a gradient of $5 \%$ buffer $\mathrm{B}\left(5 \% \mathrm{H}_{2} \mathrm{O}\right.$ and $95 \%$ ACN, with the pH adjusted to 9.8 with ammonia) for $10 \mathrm{~min}, 5-35 \%$ buffer $\mathrm{B}$ for $40 \mathrm{~min}, 35-95 \%$ buffer $\mathrm{B}$ for 1 $\mathrm{min}$ at a flow rate of $1 \mathrm{~mL} \cdot \mathrm{min}^{-1}$. Then the system was maintained for $3 \mathrm{~min}$ followed by a decrease to $5 \%$ buffer $B$ within $1 \mathrm{~min}$ and equilibration with $5 \%$ buffer $B$ for $10 \mathrm{~min}$. The elution process was monitored at $214 \mathrm{~nm}$ absorbance, and fractions were collected every $1 \mathrm{~min}$ and pooled as 20 fractions. The eluted peptides were subsequently concentrated by vacuum centrifugation.

\subsubsection{LC-MS/MS Analysis}

The fractions were resuspended in buffer $A$ ( $2 \%$ acetonitrile and $0.1 \%$ formic acid), and then centrifuged for $10 \mathrm{~min}(20,000 \times \mathrm{g})$. Supernatants were loaded on the $\mathrm{C}_{18}$ trap column on a LC-20 AD nano-HPLC instrument. An internally packed analytical $\mathrm{C}_{18}$ column was used to elute and separate the peptides. The elution was performed at a flow rate of $600 \mathrm{~nL} \cdot \mathrm{min}^{-1}$ under the following conditions: $8 \%-35 \%$ buffer $\mathrm{B}\left(5 \% \mathrm{H}_{2} \mathrm{O}\right.$ and $95 \%$ ACN, with the $\mathrm{pH}$ adjusted to 9.8 with ammonia), 0-35.00 min; $60 \%$ buffer $\mathrm{B}, 35.01-40.00 \mathrm{~min} ; 80 \%$ buffer $\mathrm{B}$, 40.01-45.00 min; 5\% buffer B, 45.01-45.10 min. The equilibration time was $10 \mathrm{~min}$. The eluted peptide was subjected to nano-electrospray ionization followed by MS/MS (Orbitrap Fusion Lumos mass spectrometer, CA, USA) and nano HPLC system analyses. The mass spectrometry analyses were performed with a scan range of $350-1800 \mathrm{~m} / \mathrm{z}$, and the survey scans were obtained at 120,000 mass resolution of $400 \mathrm{~m} / \mathrm{z}$ via Orbitrap analyzer. Dynamic exclusion parameters mainly included exclusion count of 2 and exclusion time of $30 \mathrm{~s}$.

\subsubsection{Data processing and proteins identification}

Proteome Discoverer software v2.1 (Thermo Fisher Scientific, Massachusetts, USA) was applied to process and quantify raw data files. Protein search was performed in RefSeq Human protein database (24078 sequence, released in 2017) based on SEQUEST algorithm. Parameters were set as follows: fixed modifications, including carbamidomethylation of cysteine, modification of $\mathrm{N}$ terminus and $\mathrm{K}$ of TMT sixplus. And methionine oxidation was applied as variable modification. Trypsin was set as the enzyme, allowing 2 missing cleavages. The verification and identification were performed by Percolator software (Proteome Discoverer sequest (v2.1)). The protein and peptide profile matching false discovery rate (FDR) was set at 0.01 [21]. Total protein intensity was generated by summing all reported ion intensities of unique peptides that matched each protein. Correction of Bias and Background were performed by checking protein quantification and normalization. Proteins containing at least two unique peptides were further quantified.

\subsubsection{Statistical analysis of proteomics}


For proteomic analysis, proteins were extracted from 3 biological replications in each group. The fold changes of proteins in control, model, FA and FB group were calculated as mean value according to the relative and absolute quantification ratio of the protein isobaric tags. Student's t-test of SPSS 25.0 was performed to further determine whether the differential proteins were significantly different $(p<0.05)$ between groups. Accordingly, proteins with $p$ value $<0.05$ and fold change $>1.2$ were identified as differentially expressed biomarkers.

\subsection{Interactive network construction of metabolites and proteins}

For pathway and network analyses, the correlation coefficient and $p$ value of differentially expressed metabolites and proteins (with NCBI IDs) were calculated by R language. The metabolites and proteins with $p$ $<0.05$ and absolute correlation coefficient $>0.9$ were screened out, and further imported into Cytoscape software (v3.4.0) to generate the final association analysis network diagram.

\subsection{Quantitative real-time PCR (qRT-PCR) analysis}

PCR reactions were performed to analyze the mRNA expressions of nucleoside diphosphate kinase 3 isoform X1 (Nme3), WD repeat-containing protein 3 (Wdr3), 28S ribosomal protein S7 mitochondrial precursor (Mrps7), Collagen, inflammatory mediators such as IL-6, IL-1 $\beta$, TNF- $\alpha$ and genes involved in nuclear transcription factor-kappa B (NF-KB), mitogen-activated protein kinases (MAPK) and Janus kinase/signal transducer and activator of transcription (JAK-STAT) signaling pathways. In brief, zebrafish were washed three times with RNase-free water, which were then homogenized with Trizol reagent. Homogenized tissues were extracted by chloroform and centrifuged for $15 \mathrm{~min}\left(12,000 \times \mathrm{g}, 4^{\circ} \mathrm{C}\right)$. The supernatant was transferred to a new tube and isopropyl alcohol of equal volume was added for RNA precipitation. After being centrifuged for $10 \mathrm{~min}\left(12,000 \times \mathrm{g}, 4^{\circ} \mathrm{C}\right), 75 \%$ ethanol was used to wash the pellet which was then dried and dissolved in $50 \mu \mathrm{L}$ RNase-free water. The OD260/280 value was measured for RNA purity detection. The reaction procedures were set as follows: $95^{\circ} \mathrm{C}$ for $10 \mathrm{~min}, 95^{\circ} \mathrm{C}$ for $15 \mathrm{~s}, 60^{\circ} \mathrm{C}$ for $30 \mathrm{~s} \mathrm{(40} \mathrm{cycles).} \mathrm{The} \mathrm{relative} \mathrm{mRNA}$ expressions were shown by calculating values of $2^{-\triangle \Delta C T}$. Primers of all genes for amplification were designed by Primer-BLAST (NCBI) which was listed in Table S1. For gene expression analysis, one-way ANOVA of SPSS 25.0 was used for statistical comparisons. Data were shown as mean \pm S.D and $p<0.05$ were assumed for the probability level for statistical significance.

\section{Results}

\subsection{Inhibitory effects of FA and $\mathrm{FB}$ in $\mathrm{CuSO}_{4}$-induced inflammation in zebrafish larvae}

The primary lateral line system of zebrafish was well established within 3 days after fertilization. Previous studies have reported that the addition of $\mathrm{CuSO}_{4}$ can swiftly destroy the hair cells of neuromasts in zebrafish lateral line through oxidation and cell death [22]. The zebrafish larvae of each treatment group were observed under fluorescence microscope and the number of fluorescent cells in region of about ten cell diameters within the horizontal muscle of zebrafish was counted (Fig. 2A). In control group, most of neutrophils were assembled in the posterior blood island or caudal hematopoietic tissues, an area where most leukocytes were distributed in this development stage (Fig. 2B). In contrast, zebrafish exposed to $10 \mu \mathrm{mol} \bullet \mathrm{L}^{-1} \mathrm{CuSO}_{4}$ for 40 
min formed a typical neutrophil cluster in horizontal muscles, which indicated that neutrophils actively migrated to the neuromasts of lateral line induced by $\mathrm{CuSO}_{4}$. In contrast to $\mathrm{CuSO}_{4}$ exposure, co-treatment with FA or FB inhibited neutrophils migration and decreased the assembled number of neutrophils as shown in Fig. $2 \mathrm{C}$ (the red dotted lines showed the location of the horizontal muscles). These results indicated that FA or FB can dose-dependently inhibit neutrophils recruitment to the neuromasts of zebrafish lateral line (Fig. 2D).

Fig. $2 \mathrm{FA}$ and $\mathrm{FB}$ inhibit neutrophils recruitment to the injury site in $\mathrm{CuSO}_{4}$ treated zebrafish. (A) A general view of $3 \mathrm{dpf}$ zebrafish larvae. The boxed area referred to the horizontal lateral line, and neutrophils within the horizontal line (dotted red lines) were calculated in quantification experiments. (B) The area of the posterior blood island or caudal hematopoietic tissues (the boxed region with dotted yellow lines) in untreated Transgenic (mpx:EGFP) zebrafish. Most leukocytes in this development stage were distributed in this area. (C) Microphotographs showing neutrophils migration in control, model, FA and FB group in different concentrations (neutrophils of $3 \mathrm{dpf}$ transgenic (mpx:EGFP) zebrafish exhibiting green fluorescence). The red dotted lines showed the location of the horizontal muscles of zebrafish. (D) FA and FB dose-dependently reduced the number of neutrophils counted in the injury site. ${ }^{\#} p<0.05,{ }^{\# \#} p<0.01,{ }^{\# \#} p<0.001$, compared with control group; ${ }^{*} p<0.05,{ }^{* *} p<0.01,{ }^{* \star *} p<0.001$, compared with model group. Data were shown as mean \pm S.D, $n=35$.

\subsection{Inhibitory effects of FA and $\mathrm{FB}$ against $\mathrm{CuSO}_{4}$-induced intracellular $\mathrm{ROS}$ and $\mathrm{NO}$ generation in zebrafish larvae}

The overproduction of ROS by polymorphonuclear neutrophils at inflammatory sites can cause endothelial dysfunction and tissue damage [23]. Therefore, DCF-DA was used to detect ROS generation in $\mathrm{CuSO}_{4}$-induced inflammation in zebrafish model. Fig. 3A exhibited a representative image of intracellular ROS production and inhibitory effects of FA and $\mathrm{FB}$ on ROS accumulation in zebrafish against $\mathrm{CuSO}_{4}$ exposure. The control group, not treated with $\mathrm{CuSO}_{4}$ or FA and FB, exhibited a clear and weaker fluorescent image. However, the model group exposed to $\mathrm{CuSO}_{4}$ showed a bright and strong fluorescent image. Thus, addition of $\mathrm{CuSO}_{4}$ can lead to intracellular ROS generation in zebrafish. However, FA and FB dose-dependently decreased intracellular ROS accumulation in zebrafish (Fig. 3B).

Besides, $\mathrm{NO}$ is also a common conduction molecule and plays an important role in the initiation and development of inflammation [24]. Hence, the fluorescent probe DAF-FMDA was performed to evaluate the inhibitory effects of $\mathrm{FA}$ and $\mathrm{FB}$ in $\mathrm{CuSO}_{4}$-induced intracellular $\mathrm{NO}$ production in zebrafish. As a result, zebrafish exposed to $\mathrm{CuSO}_{4}$ showed an excessive generation of $\mathrm{NO}$ (Fig. 3C). However, zebrafish treated with FA or FB showed a significant decrease of $\mathrm{NO}$ accumulation in a dose-dependent manner (Fig. 3D). These evidences indicated that $\mathrm{FA}$ and $\mathrm{FB}$ could relieve $\mathrm{CuSO}_{4}$-induced inflammatory process in zebrafish via inhibiting generation of intracellular ROS and NO.

Fig. $3 \mathrm{FA}$ and $\mathrm{FB}$ alleviated inflammation in zebrafish by inhibiting $\mathrm{CuSO}_{4}$-induced $\mathrm{ROS}$ and $\mathrm{NO}$ generation. (A) Microphotographs documenting ROS production in control, model, FA and FB group in different concentrations ( $3 \mathrm{dpf}$ wild-type $\mathrm{AB}$ strain zebrafish larvae). (B) FA and $\mathrm{FB}$ dose-dependently reduced $\mathrm{CuSO}_{4}{ }^{-}$ 
induced ROS generation. (C) Microphotographs documenting production of NO in control, model, FA and FB group in different concentrations ( 3 dpf wild-type $A B$ strain zebrafish larvae). (D) FA and FB dose-dependently reduced $\mathrm{CuSO}_{4}$-induced NO generation. ${ }^{\#} p<0.05,{ }^{\# \#} p<0.01$, ${ }^{\# \# \#} p<0.001$, compared with control group; ${ }^{*} p<$ $0.05,{ }^{* *} p<0.01,{ }^{* \star *} p<0.001$, compared with model group. Data were shown as mean \pm S.D, $n=35$.

\subsection{Metabolomics}

\subsubsection{Multivariate analyses of UPLC-MS/MS data}

UPLC-MS/MS was used to analyze all zebrafish samples in both positive and negative ion mode. Representative base peak chromatograms (BPC) of zebrafish samples from control, model, FA and FB group were obtained under optimal conditions (Fig. 4A, 4B). 15489 variables (ESI+) and 26308 variables (ESI-) could simultaneously be detected in $20 \mathrm{~min}$.

Multivariate analyses suggested good separations among control, model, FA and FB group. The principal component analysis (PCA) model and the profiles of control, model, FA and FB group showed a tendency towards separation (Fig. 4C, 4D). As a model for supervised analysis, partial least squares-discriminant analysis (PLS-DA) was performed and could further distinguish the differences in metabolites among control, model, FA and FB group. The scores plot of PLS-DA indicated that the four groups were clearly distinguished (Fig. 4E, 4F). It was found that the control and model group were obviously separated both in positive mode and negative mode, indicating a $\mathrm{CuSO}_{4}$-induced acute inflammation in zebrafish model was successfully established. FA $\left(120 \mu \mathrm{mol} \cdot \mathrm{L}^{-1}\right)$ and FB $\left(150 \mu \mathrm{mol} \cdot \mathrm{L}^{-1}\right)$ group were closer to the control group, which highlighted the anti-inflammation effects of drugs.

\subsubsection{Identification and analysis of metabolites}

The heat-maps of differential metabolites changes of control vs. model, model vs. FA, model vs. FB were shown in Fig. 4G, 4H, 4I. Identification of these metabolites was carried out based on information of MS/MS and online database. A total of 88 metabolites were found to be the most significantly altered in model group relative to control group. FA and FB reversed the change of 14 and 35 metabolic biomarkers, respectively. As listed in Table 1, 36 metabolic biomarkers involved in $\mathrm{CuSO}_{4}$-induced inflammation in zebrafish were identified in FA or FB group. Both FA and FB could reverse abnormal expressions of Uridine 5-Diphosphate, Carnitine, Val-Abu-OH, Ciliatine, Cytidine, Benzaldehyde, Isobutyryl Carnitine, N-Acetyl-L-Phenylalanine, Gamma-Glutamyl-Leucine, Dihydrojasmonic acid, Cyclic GMP, 12-Hydroxydodecanoic acid and NArachidonoyl Dihydroxypropylamine, which were partially involved in inflammatory response and suggested that FA and FB may exert anti-inflammatory effect through regulating expressions of these metabolites [2530].

Analyses of topology and pathway enrichment based on KEGG database were performed to identify the metabolomic pathways affected by these metabolites. Metaboanalyst was applied to calculate the $-\log (p)$ value and the pathway impact value from analyses of pathway enrichment and topology, respectively. Potential pathways of $\mathrm{FA}$ and $\mathrm{FB}$ in $\mathrm{CuSO}_{4}$-induced inflammation in zebrafish were finally identified according to pathway impact and $-\log (p)$ value summarized in Table S2. The identified biomarkers and related 
pathways from KEGG database were shown in Fig. 4J, which indicated that Nicotinate and nicotinamide metabolism, Energy metabolism, Pyrimidine metabolism and Purine metabolism were mainly involved in $\mathrm{CuSO}_{4}$-induced inflammation in zebrafish.

Fig. 4 Metabolomic analyses of zebrafish larvae. Representative Base Peak Chromatogram (BPC) of zebrafish in control, model, FA and FB group obtained from positive ion mode (A) and negative ion mode (B). PCA score plot of zebrafish metabolites from positive ion mode (C) and negative ion mode (D). PLS-DA score

plot of zebrafish metabolites from positive ion mode $(E)$ and negative ion mode $(F)$. Heat-map of metabolites of control vs. model (G), model vs. FA (H), model vs. FB (I). Rows: differential metabolites; Columns: zebrafish samples. The rectangle in different color represented the expression level of metabolites. Highest in red; lowest in blue; mean in white. (J) Metabolic pathways (bold) participating in the anti-inflammatory action of $\mathrm{FA}$ and $\mathrm{FB}$ in $\mathrm{CuSO}_{4}$-induced inflammation in zebrafish. The metabolites (red) were identified biomarkers in the present study. Arrows nearby metabolites indicated the relative expressions of metabolites of control vs. model, model vs. FA, model vs. FB.

\subsection{Proteomics}

\subsubsection{Proteins identification}

The proteomic profiles of $\mathrm{CuSO}_{4}$-induced inflammation in zebrafish after FA and FB treatment were analyzed by UPLC-MS/MS. As a result, 5212 proteins were detected. Among which, 2976 proteins were further identified and quantified with at least two unique peptides and false discovery rate $(F D R)<1 \%$. According to the criteria of fold change $>1.2$ and $p<0.05,146$ proteins were differentially expressed in model group compared with control group, including 60 upregulated proteins and 86 downregulated proteins. Additionally, the expressions of 51 and 18 differentially expressed proteins (DEPs) were reversed in FA and FB group, respectively (Table 2).

\subsubsection{Analyses of DEPs}

The heat-maps of differential protein changes of control vs. model, model vs. FA, model vs. FB were presented in Fig. 5A, 5B, 5C. The DEPs were categorized according to the following Gene Ontology (GO) classes: biological process, molecular function and cellular components (Fig. S1). KEGG analysis was carried out to further identify the specific biological pathways associated with $\mathrm{CuSO}_{4}$-induced inflammation in zebrafish, and further clarify the therapeutic mechanism of FA and FB. As shown in Fig. 5D, 5E and 5F, the identified proteins were involved in a variety of pathways, including Cellular Processes, Environmental Information Processing, Genetic Information Processing, Human Diseases, Metabolism and Organismal Systems, which covered a wide range of biological pathways in inflammatory response and neuromasts damage processes.

The identified DEPs were imported to STRING database (https://string-db.org/) for protein-protein interaction (PPI) network constructions (Fig. S2). Compared with control group, among the 146 identified DEPs in model group, 101 proteins were involved in the network, 40 proteins were related to each other, and 5 proteins did not display any linkage at a confidence level of string score $=0.4$. This network reflected a complex functional relationship among the proteins identified in the current study. 
Fig. 5 Proteomic analyses of zebrafish larvae. Heat-map of differential proteins of control vs. model (A), model vs. FA (B), model vs. FB (C). Rows: differential proteins; Columns: zebrafish samples. The rectangle in different color represented the expression level of proteins. Highest in red; lowest in green; mean in white. The KEGG pathway classification of differential proteins of control vs. model (D), model vs. FA (E), model vs. FB (F). Rows: number of proteins; Columns: KEGG pathway classification.

\subsection{Interactive network of identified metabolites and proteins}

The differentially expressed metabolites and proteins from groups of control vs. model, model vs. FA and model vs. FB were imported into Cytoscape software to conduct the significant network analyses (Fig. S3). Further integrated analyses of metabolomic and proteomic studies showed altered pathways in $\mathrm{CuSO}_{4}{ }^{-}$ induced acute inflammation involving Lipid metabolism, Amino acid metabolism and Nucleotide metabolism (Fig. 6A, 6B, 6C). FA and FB were likely to reverse expressions of metabolites and proteins related to these metabolic pathways, thus alleviating hair cells injury in zebrafish.

Fig. 6 Interactive network construction by integrated metabolomics and proteomics analyses. Interactive network of metabolites and proteins of model group compared with control (A), FA (B), and FB group (C). Triangles and circles in different colors represented metabolites and proteins in different metabolic pathways, respectively.

\subsection{QRT-PCR}

The mRNA levels of Wdr3, Nme3, Collagen, Mrps7, inflammatory mediators IL-6, IL-1 $\beta$, TNF-a contributing to the early inflammatory phase and mediating the recruitment of other immune cells to the damaged areas, and genes involved in NF-KB, MAPK, JAK-STAT signaling pathways in different groups were detected by qRT-PCR. As a result, gene expressions of Wdr3, Nme3, Collagen and Mrps7 in FA and FB group were decreased relative to model group, which was consistent with proteomic results (Fig. 7A). Moreover, FA and FB significantly downregulated IL-6, IL-1 $\beta$ and TNF-a expression compared with model group (Fig. 7B). FB could reverse mRNA expression of genes involved in NF-KB, MAPK and JAK/STAT signaling pathways. However, FA mainly exerted anti-inflammation effect via NF-KB and MAPK signaling pathways (Fig. 7C).

Fig. 7 Relative expressions of mRNA in different groups detected by qRT-PCR. (A) The mRNA expressions of Wdr3, Nme3, Collagen, Mrps7 of control, model, FA and FB group. (B) The mRNA expressions of IL-6, IL-1 3 , TNF-a of control, model, FA and FB group. (C) The mRNA expressions of genes involved in NF-KB, MAPK, JAKSTAT signaling pathways of control, model, FA and FB group. ${ }^{\#} p<0.05,{ }^{\# \#} p<0.01,{ }^{\# \#} p<0.001$, compared with control group; ${ }^{*} p<0.05,{ }^{* *} p<0.01,{ }^{* \star *} p<0.001$, compared with model group. Data were shown as mean \pm S.D, $n=35$.

\section{Discussion}

Inflammatory response is a common and highly regulated biological process in the body, which allows the immune system to clear harmful irritants and effectively initiate reparation [31]. In the process of inflammation, excessive recruitment and uncontrolled migration of neutrophils to injured sites could result in 
tissue damage and disease [24]. In this case, we used $\mathrm{CuSO}_{4}$ to induce inflammation in zebrafish so that the behavior of neutrophil cells in zebrafish during the critical transitions can be monitored. $\mathrm{CuSO}_{4}$ is a chemical metal that induces neuromasts damage of zebrafish lateral line system and can easily reflect the characteristics of antioxidant and pro-inflammation. In addition to regenerative capacity, hair cells in zebrafish lateral line and mammal have great similarities in structure, function and molecularity [32]. In the present study, neutrophils were significantly accumulated in the damaged neuromasts of zebrafish lateral line against $\mathrm{CuSO}_{4}$ exposure. However, the number of neutrophils in neuromasts was clearly reduced when zebrafish were treated with FA or FB, which indicated that FA and FB may exert excellent neuroprotective properties against $\mathrm{CuSO}_{4}$-induced neuroinflammation via exhibiting anti-inflammatory activities.

Excessive ROS and NO production in the body may lead to cell and tissue damage. Therefore, inhibiting the excessive production of NO and ROS can effectively inhibit the progress of inflammatory response, which can also be considered as a potential anti-inflammatory drug development strategy. Addition of $\mathrm{CuSO}_{4}$ significantly increased ROS and NO production in zebrafish. As reported, the excessive oxidative stress due to the overproduction of ROS can form a continuous stimulation of neurons and lead to neurons damage and dysfunction [33, 34]. However, FA and FB markedly inhibited ROS and NO accumulation in $\mathrm{CuSO}_{4}$-induced neuromasts damage in zebrafish, which further suggested the potential neuroprotection of FA and FB against $\mathrm{CuSO}_{4}$ exposure through their remarkable anti-inflammatory properties.

Metabolomic analysis has identified multiple metabolites between control and model group. Among which, the abnormal expressions of Uridine 5-Diphosphate, Carnitine, Val-Abu-OH, Ciliatine, Cytidine, Benzaldehyde, Isobutyryl Carnitine, N-Acetyl-L-Phenylalanine, Gamma-Glutamyl-Leucine, Dihydrojasmonic acid, Cyclic GMP, 12-Hydroxydodecanoic acid and N-Arachidonoyl Dihydroxypropylamine were all reversed both in FA and FB group. Some metabolites have been reported to participate in the modulation of particular molecules or inflammatory mediators and play a crucial role in inflammatory cascade reaction. For example, Uridine 5Diphosphate can induce the expression of chemokines such as monocyte chemotactic protein 1 (MCP-1) and macrophage inflammatory protein 1a (MIP-1a) in microglia and astrocytes, which were the primary effector molecules that mediated the recruitment of inflammatory cells to the damaged tissues $[25,35]$. Therefore, the potential role of Uridine 5-Diphosphate in inflammatory response was likely to be related to the involvement of chemokines in inflammation. The depletion of Carnitine was previously reported to be prevalent in hemodialysis patients, which was considered to be associated with inflammatory response in patients [36]. Besides, the requirement of exogenous Carnitine was demanded to satisfy energy consumption, which may reflect an indirect modulation of Carnitine in inflammatory response via regulating Energy metabolism. The specific role of Energy metabolism in inflammation will be thoroughly discussed later. Additionally, Cytidine can be metabolized to uridine via cytidine deaminase, which activated and increased nucleoside-sensing of Toll-like Receptor 8 accompanied with an overexpression of TNF-a in monocyte or myeloid cells [37, 38]. Thus, the involvement of Cytidine in inflammation may be related to the inflammatory cascade reaction induced by the activation of Toll-like Receptors.

The further pathway enrichment analysis of metabolomics indicated that Nicotinate and nicotinamide metabolism, Energy metabolism, Pyrimidine metabolism and Purine metabolism were mainly involved, most of which were directly or partly involved in inflammation [39-43]. The involvement of Nicotinate and 
nicotinamide metabolism in inflammatory response may be related to some metabolic enzymes as well as secondary metabolites produced during metabolic processes. For example, nicotinate phosphoribosyltransferase was an important inflammatory mediator and can bind with Toll-like receptor 4 , which in turn can induce the activation of inflammasomes and NF-kB for the secretion of inflammatory mediators IL-1 $\beta$, IL-8 and TNF- $\alpha$ [40]. Moreover, the identified metabolite Nicotinamide Ribotide that participated in Nicotinate and nicotinamide metabolism, also known as nicotinamide single nucleotide $(\mathrm{NMN})$, can be converted into the principal precursor of nicotinamide adenine dinucleotide $\left(N A D^{+}\right)$via transamination $[44,45]$. $\mathrm{NAD}^{+}$may regulate $\mathrm{NAD}^{+} / \mathrm{NADH}$ ratio to activate Sirtuin 1 (SIRT1), which in turn positively regulated NF-KB expression. NF-KB is an important transcription factor and involved in proinflammatory cytokines generation $[46,47]$. Presently, it can be speculated that FB might decrease NAD ${ }^{+}$ synthesis by inhibiting Nicotinamide Ribotide expression, which may further result in an inactivation of SIRT1 and NF-KB signaling pathway along with a decreased release of inflammatory factors.

Differentially expressed metabolites such as Cytidine, Cytosine, Deoxyuridine, Uracil, XMP, dIMP, Cyclic GMP and Guanosine were identified in the present study, which were all involved in Pyrimidine and Purine metabolism. It was until the participation of pyrimidine and purine nucleotide receptors (P2Y receptors) in immune function has been well explained that the involvement of Pyrimidine and Purine metabolism in inflammatory response has met a reasonable comprehension [43]. That is, P2Y receptors were presented practically in all immune cells and can specifically mediate inflammatory response for various cell injuries via recognizing pyrimidine and purine nucleotides [43]. For example, P2Y6 receptor showed the highest expression in macrophages, dendritic cells and microglia, which mainly comprised of immune phenotypes of macrophage dysfunction, microglial dysfunction, inactivation of effector $T$ cells and played a significant role in vascular inflammation [48-50]. Moreover, inhibition of P2Y6 receptor was accompanied with the decreased release of IL-8, IL-1a and IL-6 which were induced by monosodium urate crystals in human keratinocytes [51]. Therefore, the significant role of Pyrimidine and Purine metabolism in inflammatory response may be closely related to the immune functions of various $\mathrm{P} 2 \mathrm{Y}$ receptors. Consequently, it can be speculated that FA and FB might regulate these metabolites to modulate cellular immune functions and the release of various inflammatory mediators to exert anti-inflammatory effects.

At the same time, energy consumption also played an important role during the development of inflammation. In the present study, Starch and sucrose metabolism, Amino sugar and nucleotide sugar metabolism, Glycolysis, Pentose phosphate pathway (PPP) and Galactose metabolism were all related to Energy metabolism. During inflammatory response, there was an increasing requirement of energy in immune cells, which helped to satisfy the energy demand for housekeeping function and multiple specific immune cells functions, such as cell migration, cytokinesis, antigen processing and presentation and lymphocyte activation [38,52]. Inflammatory response can consume extra energy up to $25 \%-60 \%[53,54]$. As reported, the regulatory effect of tested drugs on Energy metabolism of immune cells can provide a potential treatment strategy [55,56]. For example, Glucose-6-phosphate (G6P) was the intersection of multiple energy metabolic pathways that can be converted to PPP for nicotinamide adenine dinucleotide phosphate (NADPH) and ribose-5-phosphate. Activation of PPP contributed to an inhibition of P53, which increased glucose consumption, NADPH production and biosynthesis [57]. In our study, G6P was significantly upregulated in model group relative to control group, but FB remarkably downregulated G6P, suggesting that FB may sever 
as an anti-inflammatory agent through regulating G6P expression probably along with reduced energy consumption. Meanwhile, the relieved damage in FB group may also contribute to the decreased production of G6P as well as the reduced requirement of energy consumption. That is, in $\mathrm{CuSO}_{4}$-induced acute inflammation, zebrafish needed more energy to conduct a series of inflammation-related activities such as cytokines synthesis and immune cells activation as well as to repair tissues itself. However, zebrafish in FA and FB group tended to require less energy to basically maintain for housekeeping function, that is, reduced energy consumption may be secondary to the decreased inflammatory response in FA and FB group.

The proteomic analysis has identified 146 DEPs in model group compared with control group. Among which, 51 and 18 DEPs were reversed in FA and FB group, respectively. The KEGG pathway classification of DEPs covered a variety of cellular processes and metabolic pathways that involved in inflammatory response and neuromasts damage. The DEPs widely participated in multiple biological pathways such as Immune system and Infectious disease, Nervous system and Neurodegenerative disease, Signal transduction, Cell growth and death, which suggested that both the immune and nervous system of zebrafish can be damaged due to $\mathrm{CuSO}_{4}$ exposure, involving a variety of signal transduction as well as cell death and regeneration in zebrafish. Meanwhile, the identified proteins also participated in various metabolomic pathways such as Amino acid metabolism, Lipid metabolism, Nucleotide metabolism and Energy metabolism that can influence multiple inflammation-related processes as further discussed in the integrated analyses of metabolomic and proteomic below. The identified DEPs, such as Collagen (col2a1b, col9a2, col9a1b), Wdr3, Mrps7 and Nme3 were all reversed both in FA and FB group, which were actively or passively involved in inflammatory response [58-61]. Previous studies have shown that the organizations of ROS, NF-KB and collagen at the injury margin were involved in the promotion of wound healing [62]. High levels of TNF-a may lead to excessive inflammatory response along with increased deposition of collagen in fibroblast-treated wounds [63]. In addition to the generation of collagen, remodeling of the collagen structure induced by tissue damage also played a crucial role in the repair process, which may be achieved by proteases such as matrix metalloproteinase (MMP) $[64,65]$. The important role of MMP9 in collagen reorganization and regeneration has also been reported, which suggested that MMP9 can aggravate wound damage in chronic injury, but promote wound healing and regeneration in acute injury [66]. This implied a critical role of collagen in tissue damage and repair. Our findings suggested that both FA and FB might guide therapeutics focused on damage prevention (neuroprotection) or damage repair (anti-inflammation) in $\mathrm{CuSO}_{4}$-induced hair cells wounds in zebrafish larvae via reversing the productions of Collagen.

Mitochondrial ribosomal protein (MRP) subunits were nuclear-encoded and were crucial for mitochondrial functions as well as mitochondrial encoded synthesis of proteins, some of which were also reported to be involved in mitochondrial diseases [61, 67]. Mrps7, a 12S ribosomal RNA binding subunit, was necessary for assembly of small ribosomal subunits. Studies have confirmed that Mrps7 mutations can cause mitochondrial respiratory chain dysfunction and congenital sensorineural deafness [68]. Mitochondria were an important component in innate inflammatory response and mitochondrial dysfunction was a vital trigger of inflammation, which can result in metabolic alteration and mitochondrial damage-induced release of inflammatory mediators [69]. Thus, we speculated that $\mathrm{CuSO}_{4}$ exposure induced Mrps7 alteration might be related to mitochondrial dysfunction in zebrafish, which was the main source of ROS owing to the increased energy demand and efficient mitochondrial function dependent excessive inflammatory activities [70].

Page 15/38 
As a member of WD repeat-containing proteins, Wdr3 participated in several cellular processes such as cell cycle progression and signal transduction [71, 72]. Studies have confirmed that some Wdrs participated in the transduction of MAPK and STAT3 signaling pathways [73]. Moreover, expression of misfolded Wdrs in dendritic cells can result in a substantial release of IL-18 via activating inflammasome [60]. Here we can speculate that FA and FB might reverse the abnormal expression of Wdr3 to regulate Wdrs-mediated cell cycle progression, signal pathways transduction as well as the release of inflammatory mediators and inflammasomes. Nme3, also known as NM-23, participated in a variety of physiological and pathological cellular processes such as differentiation, development, cellular signaling and cellular function. It was reported that $\mathrm{Nm} 23-\mathrm{H} 1$ could interact with p53 and positively mediated apoptosis and arrested cell cycle induced by p53 [74]. Besides, Nme3 was confirmed to modulate the pro-inflammatory transcription via activating NF-KB [75], which also contributed to Toll-like receptor 5 mediated NF-KB signaling pathway in a MyD88-dependent manner [59]. In the present study, both FA and FB could downregulate Nme3 expression, which indicated a potential regulation of cell signaling and cellular functions of FA and FB.

The integrated analyses of metabolomic and proteomic studies indicated that Lipid metabolism, Amino acid metabolism and Nucleotide metabolism were significantly altered during $\mathrm{CuSO}_{4}$ exposure, which showed a more comprehensive understanding of the metabolic pathways involved in $\mathrm{CuSO}_{4}$-induced inflammation in zebrafish. Moreover, pathways identified in metabolomics such as Pyrimidine and Purine metabolism were also indispensable pathways in Lipid metabolism, Amino acid metabolism and Nucleotide metabolism for their critical role in lipids activation, energy conservation and nucleotide synthesis [76]. Presently, lipids were considered as an effective signal molecule to regulate multiple cellular responses. A variety of lipid-derived mediators were involved in the regulation of inflammation as well as infection response [77, 78]. For example, lipid-derived mediators Prostaglandin E2 (PGE $)$ and Prostacyclin $\left(\mathrm{PGI}_{2}\right)$ functioned as vasodilator agents in the initial stage of inflammation for the recruitment of various immune cells, such as neutrophils and macrophages from the blood to the infected or damaged tissues [79]. Therefore, it can be speculated that FA and FB may exert therapeutic effects by regulating Lipid metabolism, which in turn may affect the role of lipid-derived mediators in inflammatory response. Amino acid metabolism was also considered to mediate immune response in mammals for the pivotal enzymes and the degradation of amino acids can be crucial immune checkpoints in autoimmunity [80]. The activation of enzymes such as indoleamine 2,3-dioxygenase 1 (ID01) and arginase 1 (ARG1) can induce a potential immunoregulatory effect of dendritic cells and inflammatory cells capable of coordinating immune response and resistance [81, 82]. Additionally, Nucleotide metabolism can also occur in inflammatory conditions and tissue injury [83]. The extracellular release of various nucleotides, such as ATP, ADP and UDP, can specifically bind with individual P2Y receptors, which in turn participate in tissue damage as well as the recruitment of immune cells [83-85]. Therefore, FA and FB may exhibit anti-inflammatory activities by modulating Amino acid metabolism and Nucleotide metabolism thus reducing immune response and alleviating neuromasts damage in zebrafish.

Besides, the mRNA expressions of genes involved in NF-KB, MAPK, JAK-STAT inflammatory signaling pathways and inflammatory cytokines such as IL-6, IL-1 $\beta$ and TNF- $a$ were also detected in the present study. NF-KB, MAPK and JAK-STAT pathways were widely involved in the signaling transduction of inflammatory response such as regulating various chemokines and inflammatory cytokines secretion, which contributed to an amplified inflammatory response and worse tissue damage [86-88]. Our findings suggested that FA and FB 
may ameliorate inflammation via interfering negatively with these signaling pathways. Meanwhile, IL-6, IL-1 $\beta$ and TNF-a were the most studied pro-inflammatory cytokines that contributed to the early inflammatory phase and participated in multiple inflammation-related processes, which were partly mediated by their crucial role in the recruitment of additional inflammatory cells to the infected sites or injured tissues [3]. Besides, IL-6, TNF- $\alpha$ and IL-1 $\beta$ were also reported to play a significant role in neurocyte damage induced neuropathic pain [89]. Immediately after nerve injury, neutrophils and macrophages were recruited to the injured area and resulted in a substantial release of cytokines such as IL-6, TNF- $\alpha$ and IL-1 $\beta$, which can directly regulate the activity of neurons $[89,90]$. In the present study, $\mathrm{CuSO}_{4}$ was used to specifically induce neuromasts damage in the lateral line system of zebrafish and initiated the invasion of immune cells such as neutrophils possibly along with the release of inflammatory mediators IL-6, TNF- $a$ and IL-1 $\beta$. However, both FA and FB inhibited neutrophils recruitment as well as the secretion of inflammatory mediators induced by $\mathrm{CuSO}_{4}$, thus reducing inflammation and alleviating neuromasts damage. It can be further proved that $\mathrm{FA}$ and FB may exhibit neuroprotection against $\mathrm{CuSO}_{4}$-induced neuroinflammatory response through antiinflammatory activities.

Presently, the anti-inflammatory effects and mechanisms of FA and $\mathrm{FB}$ in $\mathrm{CuSO}_{4}$-induced inflammation in zebrafish were investigated by integrated analyses of metabolomic and proteomic studies. Our results showed that both $\mathrm{FA}$ and $\mathrm{FB}$ significantly inhibited $\mathrm{CuSO}_{4}$-induced hair cells injury in neuromasts of zebrafish. Here, we speculated that FA and FB might exhibit neuroprotective properties in $\mathrm{CuSO}_{4}$-induced neuromasts damage of zebrafish, which were mainly mediated by their anti-inflammatory activities supported by reduced recruitment of neutrophils and inhibited expressions of ROS, NO and inflammatory mediators IL-6, IL-1 $\beta$ and TNF-a. Metabolomic and proteomic analyses indicated that FA and FB might share similar antiinflammation mechanism in some extent because of the same maternal nucleus structure. The different substituents of hydroxyl groups of FA and FB may show a minor difference in anti-inflammatory activities. For example, FA showed no effect on JAK/STAT signaling pathway, on the contrary, FB can reverse the overexpression of STAT3 and JAK3, which indicated that FA and FB were likely to show a minor difference in the regulation of inflammatory signaling pathways.

As a summary, we produced a brief FA and FB action diagram (Fig. 8). FA and FB administration resulted in reductions of ROS and NO, gene expressions of Wdr3, Collagen, Nme3 and Mrps7, and other inflammatory mediators induced by $\mathrm{CuSO}_{4}$, thus reducing inflammation. As discussed above, a series of metabolites, metabolic pathways, proteins and gene products were altered during $\mathrm{CuSO}_{4}$ exposure, which can be reversed after being treated by FA or FB through specific regulatory mechanisms. Our findings suggested that these identified metabolites and proteins may sever as potential anti-inflammatory biomarkers and FA and FB exerted excellent anti-inflammatory activities in $\mathrm{CuSO}_{4}$-induced inflammation in zebrafish. Since it was a multi-component and multi-regulatory process, FA and FB might influence the downstream conduction process by affecting some inflammatory process upstream. For instance, reductions in NO and ROS may lead to secondary effects on their downstream products, such as a decreased expression of downstream inflammatory mediators IL-6, IL-1 $\beta$ and TNF- $\alpha$ via modulating specific inflammatory signaling pathways. Accordingly, the reversed expressions of Collagen, Nme3, Wdr3 and Mrps7 in FA and FB group against $\mathrm{CuSO}_{4}$ exposure may also be secondary to the regulatory effects of some metabolic pathways upstream. In addition, 
the fact that zebrafish were both pre- and post-treated with FA or FB may provide another potential therapeutic mechanism that pretreatment may attenuate the initiation of inflammation wherein posttreatment may inhibit the inflammatory cascade as well. Both administration method will inhibit inflammation through different mechanisms with different implications as a therapeutic, which needed to be further studied in the future.

Fig. $8 \mathrm{~A}$ probable mechanism of $\mathrm{FA}$ and $\mathrm{FB}$ in the protection of $\mathrm{CuSO}_{4}$-induced inflammation in zebrafish. $\mathrm{FA}$ and FB administration resulted in reductions of ROS and NO, alterations of various metabolites, proteins and gene products induced by $\mathrm{CuSO}_{4}$, thus reducing inflammation. This diagram showed the multi-component and multi-regulatory therapeutic mechanism of $\mathrm{FA}$ and $\mathrm{FB}$ against $\mathrm{CuSO}_{4}$-induced inflammation in zebrafish.

\section{Conclusion}

In conclusion, we analyzed the inhibitory effect and therapeutic mechanism of $\mathrm{FA}$ and $\mathrm{FB}$ in $\mathrm{CuSO}_{4}$-induced inflammation in zebrafish using UPLC-MS/MS based metabolomics and TMT-6 Plex based proteomics for the first time. A series of metabolic intermediates and proteins were altered during the development of inflammation. These biomarkers may clarify the anti-inflammatory mechanism of $\mathrm{FA}$ and $\mathrm{FB}$ in $\mathrm{CuSO}_{4^{-}}$ induced acute inflammation in zebrafish. These findings indicated a potential novel and sequential mechanism for the initiation of $\mathrm{CuSO}_{4}$-induced inflammation in zebrafish and suggested a multi-component and multi-regulatory therapeutic mechanism of FA and FB.

\section{Additional File Information}

Additional file 1: Table S1. Primers used for quantitative real-time PCR.

Additional file 2: Table S2. Metabolic pathways associated to varied influences of $\mathrm{FA}$ and $\mathrm{FB}$ in $\mathrm{CuSO}_{4^{-}}$ induced zebrafish inflammation.

Additional file 3: Fig. S1. GO analysis of differentially expressed proteins.

Additional file 4: Fig. S2. Protein-protein interactions of differentially expressed proteins.

Additional file 5: Fig. S3. Interactive network construction by integrated metabolomics and proteomics analysis.

\section{Abbreviations}

FA, Forsythiaside A; FB, Forsythiaside B; IL-6, Interleukin-6; TNF-a, Tumor necrosis factor-a; IL-1ß, Interleukin$1 \beta$; dpf, days postfertilization; DCF-DA, 2,7-dichlorodihydrofluorescein diacetate; DAF-FMDA, diamino fluorophore 4-amino-5-methylamino-2',7'-difluorofluorescein diacetate; ROS, reactive oxygen species; NO, nitric oxide; LC-MS, Liquid Chromatography-Mass Spectrometry; HPLC, High-Performance Liquid Chromatography; ESI-MSn, electrospray ionization mass detection; $\mathrm{m} / \mathrm{z}$, mass to charge ratio; MS/MS, Mass Spectrometry/Mass Spectrometry; HCD, higher energy collisional dissociation; UPLC-MS/MS, Ultra- 
Performance Liquid Chromatography-Mass Spectrometry/Mass Spectrometry; OPLS-DA, orthogonal projections to latent structures discriminant analysis; VIP, variable importance in the projection; ppm, part per million; HMDB, Human Metabolome Database; KEGG, Kyoto Encyclopedia of Genes and Genomes Database; TMT, Tandem Mass Tags; RP, reverse phase; NF-KB, nuclear transcription factor-kappa B; MAPK, mitogenactivated protein kinases; JAK-STAT, Janus kinase/signal transducer and activator of transcription; BPC, Base Peak Chromatogram; PCA, principal component analysis; PLS-DA, partial least squares-discriminant analysis; DEPs, differentially expressed proteins; FDR, false discovery rate; qRT-PCR, quantitative real-time PCR; GO, Gene Ontology; PPI, Protein-Protein Interaction; MCP-1, monocyte chemotactic protein 1; MIP-1a, macrophage inflammatory protein $1 a ; N M N$, nicotinamide single nucleotide; $N A D+$, nicotinamide adenine dinucleotide; NADH, nicotinamide adenine dinucleotide; SIRT1, Sirtuin 1; P2Y, pyrimidine and purine nucleotide; PPP, Pentose phosphate pathway; G6P, Glucose-6-phosphate; NADPH, nicotinamide adenine dinucleotide phosphate; MMP, matrix metalloproteinase; MRP, mitochondrial ribosomal protein; ID01, indoleamine 2,3dioxygenase 1; $A R G 1$, arginase 1; $\mathrm{PGE}_{2}$, Prostaglandin E2; $\mathrm{PGI}_{2}$, Prostacyclin.

\section{Declarations}

\section{Ethics approval and consent to participate}

All experiments were performed consistent with animal use in science in European Communities Council Directive (2010/63/EU) and followed by Chengdu University of Traditional Chinese Medicine of Laboratory Animal Ethics Committee.

\section{Consent for publication}

Not applicable.

\section{Availability of data and materials}

The datasets generated and/or analyzed in the current study are available from the corresponding author on reasonable request.

\section{Competing interests}

The authors declare no conflicts of interest.

\section{Funding}

The study was supported by National Natural Science Foundation of China (No:81373943, 81573583).

\section{Author contributions}

L.G. and Y.L. conceived and designed the experiments. L.G., L.Y., X.G., C.W., N.H. and X.D conducted the biological experiments and analyzed the data. L.G. and Y.L. wrote the manuscript. All the authors read and approved the final manuscript.

\section{Acknowledgements}


We thank Dr. Mei-chen Liu for her critical revision of the manuscript for important metabolic pathway analysis content.

\section{Studies involving human participants, human data, or human tissue}

Not applicable.

\section{References}

[1] Amor S, Peferoen LA, Vogel DY, Breur M, Valk P, Baker D, et al. Inflammation in neurodegenerative diseases-an update. Immunology. 2014;142(2):151-166.

[2] Freire MO, Van Dyke TE. Natural resolution of inflammation. Periodontol 2000. 2013;63(1):149-164.

[3] Muralidharan S, Mandrekar P. Cellular stress response and innate immune signaling: integrating pathways in host defense and inflammation. J Leukocyte Biol. 2013;94(6):1167-1184.

[4] Chen HY, Lin YH, Huang JW, Chen YC. Chinese herbal medicine network and core treatments for allergic skin diseases: implications from a nationwide database. J Ethnopharmacol. 2015;168:260-267.

[5] Pan CW, Zhou GY, Chen WL. Protective effect of forsythiaside a on lipopolysaccharide/d-galactosamineinduced liver injury. Int Immunopharmacol. 2015;26(1):80-85.

[6] Jiang WL, Fu FH, Xu BM, Tian JW, Zhu HB, Hou J. Cardioprotection with forsythoside b in rat myocardial ischemia-reperfusion injury: relation to inflammation response. Phytomedicine. 2010;17(8-9):635-639.

[7] MacRae CA, Peterson RT. Zebrafish as tools for drug discovery. Nat Rev Drug Discov. 2015;14(10):721731.

[8] Dooley K, Zon LI. Zebrafish: a model system for the study of human disease. Curr Opin Genet Dev. 2000;10(3):252-256.

[9] García-Moreno D, Tyrkalska SD, Valera-Pérez A, Gómez-Abenza E, Pérez-Oliva AB, Mulero V. The zebrafish: A research model to understand the evolution of vertebrate immunity. Fish Shellfish Immunol. 2019;90:215222.

[10] Lee SH, Ko Cl, Jee Y, Jeong Y, Jeon YJ. Anti-inflammatory effect of fucoidan extracted from ecklonia cava in zebrafish model. Carbohyd Polym. 2013;92(1):84-89.

[11] Meeker ND, Trede NS. Immunology and zebrafish: spawning new models of human disease. Dev Comp Immunol. 2008;32(7):0-757.

[12] Ghysen A, Dambly-Chaudière C. Development of the zebrafish lateral line. Curr Opin Neurobiol. 2004;14(1):67-73. 
[13] d'Alençon CA, Peña OA, Wittmann C, Gallardo VE, Jones RA, Loosli F, et al. A high-throughput chemically induced inflammation assay in zebrafish. BMC Biol. 2010;8:1-16.

[14] Kimmel CB, Ballard WW, Kimmel SR, Ullmann B, Schilling TF. Stages of embryonic development of the zebrafish. Dev Dyn. 1995;203(3):253-310.

[15] Haffter $P$, Granato M, Brand M, Mullins MC, Hammerschmidt M, Kane DA, et al. The identification of genes with unique and essential functions in the development of the zebrafish, Danio rerio. Development. 1996;123:1-36.

[16] Yang Y, Zheng K, Mei W, Wang Y, Hu J. Anti-inflammatory and proresolution activities of bergapten isolated from the roots of ficus hirta in an in vivo zebrafish model. Biochemical and Biophysical Research Communications. 2018;496(2):763-769.

[17] Trygg J, Wold S. Orthogonal projections to latent structures (O-PLS). J Chemometrics. 2002;16:119-128.

[18] Wang JB, Pu SB, Sun Y, Li ZF, Niu M, Yan XZ, et al. Metabolomic Profiling of Autoimmune Hepatitis: The Diagnostic Utility of Nuclear Magnetic Resonance Spectroscopy. J Proteome Res. 2014;13(8):3792-3801.

[19] Unwin RD, Griffiths JR, Whetton AD. Simultaneous analysis of relative protein expression levels across multiple samples using itraq isobaric tags with $2 \mathrm{~d}$ nano lc-ms/ms. Nat Protoc. 2010;5(9):1574-1582.

[20] Crowell AM, Wall MJ, Doucette AA. Maximizing recovery of water-soluble proteins through acetone precipitation. Anal Chim Acta. 2013;796:48-54.

[21] Käll L, Canterbury JD, Weston J, Noble WS, Maccoss MJ. Semi-supervised learning for peptide identification from shotgun proteomics datasets. Nat Methods. 2007;4(11):923-925.

[22] Olivari FA, Hernández PP, Allende ML. Acute copper exposure induces oxidative stress and cell death in lateral line hair cells of zebrafish larvae. Brain Res. 2008;1244:1-12.

[23] Mills EL, O'Neill LA. Reprogramming mitochondrial metabolism in macrophages as an anti-inflammatory signal. Eur J Immunol. 2016;46(1):13-21.

[24] Tripathi P, Tripathi P, Kashyap L, Singh V. The role of nitric oxide in inflammatory reactions. Fems Immunol Med Mic. 2007;51(3):443-452.

[25] Kim B, Jeong HK, Kim JH, Lee SY, Jou I, Joe EH. Uridine 5'-diphosphate induces chemokine expression in microglia and astrocytes through activation of the P2Y6 receptor. J Immunol. 2011;186(6):3701-3709.

[26] Shimizu T, Marusawa H, Endo Y, Chiba T. Inflammation-mediated genomic instability: roles of activationinduced cytidine deaminase in carcinogenesis. Cancer Sci. 2012;103(7):1201-1206.

[27] Khalatbari-Soltani S, Tabibi H. Inflammation and L-carnitine therapy in hemodialysis patients: a review. Clin Exp Nephrol. 2015;19(3):331-335. 
[28] Millet P, Opiekun M, Martin T, Beauchamp GK, Kimball BA. Cytokine contributions to alterations of the volatile metabolome induced by inflammation. Brain Behav Immun. 2018;69:312-320.

[29] Li QJ, Wang ZG, Xie Y, Liu Q, Hu HL, Gao YX. Mechanistic evaluation of gastro-protective effects of KangFuXinYe on indomethacin-induced gastric damage in rats. Chin J Nat Med. 2020;18(1):47-56.

[30] Wu J, Sun L, Chen X, Du F, Shi H, Chen C, et al. Cyclic GMP-AMP is an endogenous second messenger in innate immune signaling by cytosolic DNA. Science. 2013;339(6121):826-830.

[31] Gernez Y, Tirouvanziam R, Chanez P. Neutrophils in chronic inflammatory airway diseases: can we target them and how? Eur Respir J. 2010;35(3):467-469.

[32] Christine DC, Dora S, Fabien S, Kelly D, Nicolas G, Alain G. The lateral line of zebrafish: a model system for the analysis of morphogenesis and neural development in vertebrates. Biol Cell. 2003;95(9):579-587.

[33] de Oliveira DM, Ferreira Lima RM, El-Bachá RS. Brain rust: recent discoveries on the role of oxidative stress in neurodegenerative diseases. Nutr Neurosci. 2012;15(3):94-102.

[34] Wang J, Song Y, Chen Z, Leng SX. Connection between Systemic Inflammation and Neuroinflammation Underlies Neuroprotective Mechanism of Several Phytochemicals in Neurodegenerative Diseases. Oxid Med Cell Longev. 2018;2018:1972714.

[35] Fernandez EJ, Lolis E. Structure, function, and inhibition of chemokines. Annu Rev Pharmacol Toxicol. 2002;42:469-499.

[36] Csiky B, Bene J, Wittmann I, Sulyok E, Melegh B. Effect of hemodialysis session on the dynamics of carnitine ester profile changes in L-carnitine pretreated end-stage renal disease patients. Int Urol Nephrol. 2013;45(3):847-855.

[37] Micozzi D, Carpi FM, Pucciarelli S, Polzonetti V, Polidori P, Vilar S, et al. Human cytidine deaminase: a biochemical characterization of its naturally occurring variants. Int J Biol Macromol. 2014;63:64-74.

[38] Furusho K, Shibata T, Sato R, Fukui R, Motoi Y, Zhang Y, et al. Cytidine deaminase enables Toll-like receptor 8 activation by cytidine or its analogs. Int Immunol. 2019;31(3):167-173.

[39] Ma Y, Bao Y, Wang S, Li T, Chang X, Yang G, et al. Anti-Inflammation Effects and Potential Mechanism of Saikosaponins by Regulating Nicotinate and Nicotinamide Metabolism and Arachidonic Acid Metabolism. Inflammation. 2016;39(4):1453-1461.

[40] Managò A, Audrito V, Mazzola F, Sorci L, Gaudino F, Gizzi K, et al. Extracellular nicotinate phosphoribosyltransferase binds Toll like receptor 4 and mediates inflammation. Nat Commun. 2019;10(1):4116.

[41] Chokesuwattanaskul S, Phelan MM, Edwards SW, Wright HL. A robust intracellular metabolite extraction protocol for human neutrophil metabolic profiling. PLoS One. 2018;13(12):e0209270. 
[42] Buttgereit F, Burmester GR, Brand MD. Bioenergetics of immune functions: fundamental and therapeutic aspects. Immunol Today. 2000;21(4):192-199.

[43] Le Duc D, Schulz A, Lede V, Schulze A, Thor D, Brüser A, et al. P2Y Receptors in Immune Response and Inflammation. Adv Immunol. 2017;136:85-121.

[44] Penberthy WT. Editorial [hot topic: nicotinamide adenine dinucleotide biology and disease (executive editor: w. todd penberthy)]. Curr Pharm Design. 2009;15(1):1-2.

[45] Zhai RG, Rizzi M, Garavaglia S. Nicotinamide/nicotinic acid mononucleotide adenylyltransferase, new insights into an ancient enzyme. Cell Mol Life Sci. 2009;66(17):2805-2818.

[46] Matsushita T, Sasaki H, Takayama K, Ishida K, Kuroda R. The overexpression of sirt1 inhibited osteoarthritic gene expression changes induced by interleukin-1 in human chondrocytes. J Orthop Res. 2013;31(4):531-537.

[47] Imai SI. The nad world: a new systemic regulatory network for metabolism and aging-sirt1, systemic nad biosynthesis, and their importance. Cell Biochem Biophys. 2009;53(2):65-74.

[48] Bar I, Guns PJ, Metallo J, Cammarata D, Wilkin F, Boeynams JM, et al. Knockout mice reveal a role for P2Y6 receptor in macrophages, endothelial cells, and vascular smooth muscle cells. Mol Pharmacol. 2008;74(3):777-784.

[49] Giannattasio G, Ohta S, Boyce JR, Xing W, Balestrieri B, Boyce JA. The purinergic G protein-coupled receptor 6 inhibits effector $T$ cell activation in allergic pulmonary inflammation. $J$ Immunol. 2011;187(3):1486-1495.

[50] Riegel AK, Faigle M, Zug S, Rosenberger P, Robaye B, Boeynaems JM, et al. Selective induction of endothelial P2Y6 nucleotide receptor promotes vascular inflammation. Blood. 2011;117(8):2548-2555.

[51] Uratsuji H, Tada Y, Kawashima T, Kamata M, Hau CS, Asano Y, et al. P2Y6 receptor signaling pathway mediates inflammatory responses induced by monosodium urate crystals. J Immunol. 2012;188(1):436-444.

[52] Besedovsky HO, del Rey A. Immune-neuro-endocrine interactions: facts and hypotheses. Endocr Rev. 1996;17(1):64-102.

[53] Straub RH, Cutolo M, Buttgereit F, Pongratz G. Energy regulation and neuroendocrine-immune control in chronic inflammatory diseases. J Intern Med. 2010;267(6):543-560.

[54] Torine IJ, Denne SC, Wright-Coltart S, Leitch C. Effect of late-onset sepsis on energy expenditure in extremely premature infants. Pediatr Res. 2007;61(5 Pt 1):600-603.

[55] Seki M, Ushiyama C, Seta N, Abe K, Fukazawa T, Asakawa J, et al. Apoptosis of lymphocytes induced by glucocorticoids and relationship to therapeutic efficacy in patients with systemic lupus erythematosus. Arthritis Rheum. 1998;41(5):823-830. 
[56] Buttgereit F, Brink I, Thiele B, Burmester GR, Hiepe F, Hall ED. Effects of methylprednisolone and 21aminosteroids on mitogen-induced interleukin- 6 and tumor necrosis factor-alpha production in human peripheral blood mononuclear cells. J Pharmacol Exp Ther. 1995;275(2):850-853.

[57] Jiang P, Du W, Wang X, Mancuso A, Gao X, Wu M, et al. P53 regulates biosynthesis through direct inactivation of glucose-6-phosphate dehydrogenase. Nat Cell Biol. 2011;13(3):310-316.

[58] Cui SJ, Fu Y, Liu Y, Kou XX, Zhang JN, Gan YH, et al. Chronic inflammation deteriorates structure and function of collagen fibril in rat temporomandibular joint disc. Int J Oral Sci. 2019;11(1):2.

[59] Flentie K, Gonzalez C, Kocher B, Wang Y, Zhu H, Marasa J, et al. Nucleoside Diphosphate Kinase-3 (NME3) Enhances TLR5-Induced NFkB Activation. Mol Cancer Res. 2018;16(6):986-999.

[60] Pfajfer L, Mair NK, Heredia RJ, Genel F, Gulez N, Ardeniz Ömür, et al. Mutations affecting the actin regulator WD repeat-containing protein 1 lead to aberrant lymphoid immunity. J Allergy Clin Immunol. 2018;142(5):1589-1604.

[61] Li HB, Wang RX, Jiang HB, Zhang ED, Tan JQ, Xu HZ, et al. Mitochondrial Ribosomal Protein L10 Associates with Cyclin B1/Cdk1 Activity and Mitochondrial Function. DNA Cell Biol. 2016;35(11):680-690.

[62] LeBert D, Squirrell JM, Freisinger C, Rindy J, Golenberg N, Frecentese G, et al. Damage-induced reactive oxygen species regulate vimentin and dynamic collagen-based projections to mediate wound repair. Elife. 2018;7:e30703.

[63] Bodnar RJ, Yang T, Rigatti LH, Liu F, Evdokiou A, Kathju S, et al. Pericytes reduce inflammation and collagen deposition in acute wounds. Cytotherapy. 2018;20(8):1046-1060.

[64] Cheng F, Shen Y, Mohanasundaram P, Lindström M, Ivaska J, Ny T, Eriksson JE. Vimentin coordinates fibroblast proliferation and keratinocyte differentiation in wound healing via tgf- $\beta$-slug signaling. Proc Natl Acad Sci USA. 2016;113(30):4320-4327.

[65] Eckes B, Colucciguyon E, Smola H, Nodder S, Babinet C, Krieg T, et al. Impaired wound healing in embryonic and adult mice lacking vimentin. J Cell Sci. 2000;113(13):2455-2462.

[66] Lebert DC, Squirrell JM, Rindy J, Broadbridge E, Lui Y, Zakrzewska A, et al. Matrix metalloproteinase 9 modulates collagen matrices and wound repair. Development. 2015;142(12):2136-2146.

[67] Brodersen DE, Nissen P. The social life of ribosomal proteins. FEBS J. 2005;272(9):2098-2108.

[68] Menezes MJ, Guo Y, Zhang J, Riley LG, Cooper ST, Thorburn DR, et al. Mutation in mitochondrial ribosomal protein s7 (mrps7) causes congenital sensorineural deafness, progressive hepatic and renal failure and lactic acidemia. Hum Mol Genet. 2015;24(8):2297-2307.

[69] West AP. Mitochondrial dysfunction as a trigger of innate immune responses and inflammation. Toxicology. 2017;391:54-63. 
[70] de Mello AH, Costa AB, Engel JDG, Rezin GT. Mitochondrial dysfunction in obesity. Life Sci. 2018;192:2632 .

[71] Van der Voorn L, Ploegh HL. The wd-40 repeat. Febs Lett. 1992; 307(2): 131-134.

[72] Smith TF, Gaitatzes C, Saxena K, Never EJ. The wd repeat: a common architecture for diverse functions. Trends Biochem Sci. 1999;24(5):181.

[73] Zhu Y, Wang Y, Xia C, Li D, Li Y, Zeng W, et al. Wdr26: a novel gß-like protein, suppresses mapk signaling pathway. J Cell Biochem. 2004;93(3):579-587.

[74] Boissan M, Dabernat S, Peuchant E, Schlattner U, Lascu I, Lacombe ML. The mammalian nm23/ndpk family: from metastasis control to cilia movement. Mol Cell Biochem. 2009;329(1-2):51-62.

[75] Amendola R, Martinez R, Negroni A, Tanno B, Calabretta B, Raschellà G. DR-nm23 gene expression in neuroblastoma cells: relationship to integrin expression, adhesion characteristics, and differentiation. $\mathrm{J}$ Natl Cancer Inst. 1997;89(17):1300-1310.

[76] Chitrakar I, Kim-Holzapfel DM, Zhou W, French JB. Higher order structures in purine and pyrimidine metabolism. J Struct Biol. 2017;197(3):354-364.

[77] Zhang C, Wang K, Yang L, Liu R, Chu Y, Qin X, et al. Lipid metabolism in inflammation-related diseases. Analyst. 2018;143(19):4526-4536.

[78] Bennett M, Gilroy DW. Lipid Mediators in Inflammation. Microbiol Spectr. 2016;4(6):10.

[79] Wallace JL. Prostaglandin biology in inflammatory bowel disease. Gastroenterol Clin North Am. 2001;30(4):971-980.

[80] Mondanelli G, lacono A, Carvalho A, Orabona C, Volpi C, Pallotta MT, et al. Amino acid metabolism as drug target in autoimmune diseases. Autoimmun Rev. 2019;18(4):334-348.

[81] Mondanelli G, Bianchi R, Pallotta MT, Orabona C, Albini E, lacono A, et al. A Relay Pathway between Arginine and Tryptophan Metabolism Confers Immunosuppressive Properties on Dendritic Cells. Immunity. 2017;46(2):233-244.

[82] Banchereau J, Steinman RM. Dendritic cells and the control of immunity. Nature. 1998:392:245-252.

[83] Idzko M, Ferrari D, Eltzschig HK. Nucleotide signalling during inflammation. Nature. 2014;509(7500):310317.

[84] Myrtek D, Idzko M. Chemotactic activity of extracellular nucleotideson human immune cells. Purinergic Signal. 2007;3(1-2):5-11.

[85] Ferrari D, la Sala A, Panther E, Norgauer J, Di Virgilio F, Idzko M. Activation of human eosinophils via P2 receptors: novel findings and future perspectives. J Leukoc Biol. 2006;79(1):7-15. 
[86] Hayden MS, Ghosh S. NF-KB in immunobiology. Cell Res. 2011;21(2):223-244.

[87] Thalhamer T, McGrath MA, Harnett MM. MAPKs and their relevance to arthritis and inflammation. Rheumatology (Oxford). 2008;47(4):409-414.

[88] Banerjee S, Biehl A, Gadina M, Hasni S, Schwartz DM. JAK-STAT Signaling as a Target for Inflammatory and Autoimmune Diseases: Current and Future Prospects. Drugs. 2017;77(5):521-546.

[89] Scholz J, Woolf CJ. The neuropathic pain triad: neurons, immune cells and glia. Nat Neurosci. 2007;10(11):1361-1368.

[90] Wolf G, Gabay E, Tal M, Yirmiya R, Shavit Y. Genetic impairment of interleukin-1 signaling attenuates neuropathic pain, autotomy, and spontaneous ectopic neuronal activity, following nerve injury in mice. Pain. 2006;120(3):315-324.

\section{Tables}

Table 1 Identification results and change trends of important differential metabolites 


\begin{tabular}{|c|c|c|c|c|c|c|c|c|c|c|}
\hline No. & Metabolities & $\mathrm{m} / \mathrm{z}$ & $\mathrm{Rt} / \mathrm{s}$ & $\begin{array}{c}\text { ESI } \\
\text { mode }\end{array}$ & Formula & VIP & $\begin{array}{c}\text { P- } \\
\text { value }\end{array}$ & $\begin{array}{c}\text { Control } \\
\text { vs } \\
\text { Model }\end{array}$ & $\begin{array}{l}\text { Model } \\
\text { vs FA }\end{array}$ & $\begin{array}{l}\text { Model } \\
\text { vs FB }\end{array}$ \\
\hline 1 & Uridine 5-Diphosphate & 402.99 & 71.91 & - & $\mathrm{C}_{9} \mathrm{H}_{14} \mathrm{~N}_{2} \mathrm{O}_{12} \mathrm{P}_{2}$ & 1.793 & 0.001 & $\uparrow^{\# \# \#}$ & $\downarrow^{* *}$ & $\downarrow^{* * *}$ \\
\hline 2 & XMP & 363.03 & 75.89 & - & $\mathrm{C}_{10} \mathrm{H}_{13} \mathrm{~N}_{4} \mathrm{O}_{9} \mathrm{P}$ & 2.133 & 0.000 & $\uparrow^{\# \# \#}$ & - & $\downarrow^{* * *}$ \\
\hline 3 & Pipecolic acid & 130.08 & 78.20 & + & $\mathrm{C}_{6} \mathrm{H}_{11} \mathrm{NO}_{2}$ & 1.242 & 0.034 & $\uparrow^{\#}$ & - & $\downarrow^{*}$ \\
\hline 4 & Carnitine & 162.11 & 91.92 & + & $\mathrm{C}_{7} \mathrm{H}_{16} \mathrm{NO}_{3}$ & 1.288 & 0.027 & $\uparrow^{\#}$ & $\downarrow^{*}$ & $\downarrow^{*}$ \\
\hline 5 & 5-Methoxyindoleacetate & 206.08 & 97.77 & + & $\mathrm{C}_{11} \mathrm{H}_{11} \mathrm{NO}_{3}$ & 1.728 & 0.001 & $\uparrow^{\# \#}$ & - & $\downarrow^{*}$ \\
\hline 6 & Cytosine & 112.05 & 101.80 & + & $\mathrm{C}_{4} \mathrm{H}_{5} \mathrm{~N}_{3} \mathrm{O}$ & 1.295 & 0.026 & $\uparrow^{\#}$ & - & $\downarrow^{*}$ \\
\hline 7 & Nicotinamide Ribotide & 335.06 & 102.13 & + & $\mathrm{C}_{11} \mathrm{H}_{15} \mathrm{~N}_{2} \mathrm{O}_{8} \mathrm{P}$ & 1.990 & 0.000 & $\uparrow^{\# \# \#}$ & - & $\downarrow^{* * *}$ \\
\hline 8 & Trigonelline & 138.05 & 102.30 & + & $\mathrm{C}_{7} \mathrm{H}_{7} \mathrm{NO}_{2}$ & 1.592 & 0.004 & $\uparrow^{\# \#}$ & - & $\downarrow^{*}$ \\
\hline 9 & $\begin{array}{c}1- \\
\text { Aminocyclohexanecarboxylic } \\
\text { acid }\end{array}$ & 144.10 & 102.86 & + & $\mathrm{C}_{7} \mathrm{H}_{13} \mathrm{NO}_{2}$ & 1.787 & 0.001 & $\uparrow^{\# \# \#}$ & - & $\downarrow^{*}$ \\
\hline 10 & N-Alpha-Acetyllysine & 189.12 & 104.90 & + & $\mathrm{C}_{8} \mathrm{H}_{16} \mathrm{~N}_{2} \mathrm{O}_{3}$ & 1.275 & 0.028 & $\uparrow^{\#}$ & - & $\downarrow^{*}$ \\
\hline 11 & Val-Abu-OH & 309.11 & 105.87 & - & $\mathrm{C}_{14} \mathrm{H}_{18} \mathrm{~N}_{2} \mathrm{O}_{6}$ & 1.655 & 0.003 & $\uparrow^{\# \#}$ & $\downarrow^{*}$ & $\downarrow^{* *}$ \\
\hline 12 & Chitobiose & 425.17 & 107.48 & + & $\mathrm{C}_{16} \mathrm{H}_{28} \mathrm{~N}_{2} \mathrm{O}_{11}$ & 1.940 & 0.000 & $\uparrow^{\# \# \#}$ & $\downarrow^{* *}$ & - \\
\hline 13 & Glutamylthreonine & 249.11 & 110.44 & + & $\mathrm{C}_{9} \mathrm{H}_{16} \mathrm{~N}_{2} \mathrm{O}_{6}$ & 1.477 & 0.009 & 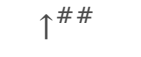 & - & $\downarrow^{* * *}$ \\
\hline 14 & Glucose 6-Phosphate & 261.03 & 112.61 & + & $\mathrm{C}_{6} \mathrm{H}_{13} \mathrm{O}_{9} \mathrm{P}$ & 1.458 & 0.000 & $\uparrow^{\#}$ & - & $\downarrow^{* * *}$ \\
\hline 15 & dIMP & 331.04 & 115.74 & - & $\mathrm{C}_{10} \mathrm{H}_{13} \mathrm{~N}_{4} \mathrm{O}_{7} \mathrm{P}$ & 1.280 & 0.029 & $\downarrow^{\#}$ & - & $\uparrow^{* *}$ \\
\hline 16 & Ciliatine & 126.03 & 118.96 & + & $\mathrm{C}_{2} \mathrm{H}_{8} \mathrm{NO}_{3} \mathrm{P}$ & 1.180 & 0.000 & $\uparrow^{\# \# \#}$ & $\downarrow^{* * *}$ & $\downarrow^{* * *}$ \\
\hline 17 & N6-Acetyl-L-Lysine & 189.12 & 121.39 & + & $\mathrm{C}_{8} \mathrm{H}_{16} \mathrm{~N}_{2} \mathrm{O}_{3}$ & 1.193 & 0.042 & $\uparrow^{\#}$ & - & $\downarrow^{*}$ \\
\hline 18 & N-A-Acetyl-L-Arginine & 217.13 & 121.98 & + & $\mathrm{C}_{8} \mathrm{H}_{16} \mathrm{~N}_{4} \mathrm{O}_{3}$ & 1.751 & 0.001 & $\uparrow^{\# \#}$ & - & $\downarrow^{* *}$ \\
\hline 19 & Uracil & 113.03 & 139.01 & + & $\mathrm{C}_{4} \mathrm{H}_{4} \mathrm{~N}_{2} \mathrm{O}_{2}$ & 1.645 & 0.003 & $\downarrow^{\# \#}$ & - & $\uparrow^{*}$ \\
\hline 20 & Cytidine & 242.08 & 174.43 & - & $\mathrm{C}_{9} \mathrm{H}_{13} \mathrm{~N}_{3} \mathrm{O}_{5}$ & 1.464 & 0.011 & $\uparrow^{\#}$ & $\downarrow^{*}$ & $\downarrow^{* *}$ \\
\hline 21 & Guanosine & 282.08 & 230.23 & - & $\mathrm{C}_{10} \mathrm{H}_{13} \mathrm{~N}_{5} \mathrm{O}_{5}$ & 1.399 & 0.015 & $\uparrow^{\#}$ & - & $\downarrow^{* * *}$ \\
\hline
\end{tabular}


$164.07 \quad 336.67+$

L-Beta-Homomethionine

279.1

(3)

337.22

$+$

$\mathrm{C}_{10} \mathrm{H}_{19} \mathrm{~N}_{3} \mathrm{O}_{4} \mathrm{~S}$

2.147 Methionine

25

Benzaldehyde

107.05

343.55

$+$

$\mathrm{C}_{7} \mathrm{H}_{6} \mathrm{O}$

$1.794 \quad 0.001$

$232.15 \quad 373.56$

$+$

$\mathrm{C}_{11} \mathrm{H}_{21} \mathrm{NO}_{4}$

$1.208 \quad 0.040$

26 Isobutyryl Carnitine

27

N-Acetyl-L-Phenylalanine

$206.08 \quad 385.01$

$-$

$\mathrm{C}_{11} \mathrm{H}_{13} \mathrm{NO}_{3}$

$1.590 \quad 0.005$

28

Gamma-Glutamyl-Leucine

$261.14 \quad 404.26$

$+$

29

Jasmonate

$209.12 \quad 521.42$

$-$

247.1

543.58

$+$

$\mathrm{C}_{11} \mathrm{H}_{21} \mathrm{~N}_{3} \mathrm{O}_{4}$

$1.904 \quad 0.000$

个\#\#\#

$\mathrm{C}_{12} \mathrm{H}_{18} \mathrm{O}_{3}$

1.0450 .000

$30 \quad$ N-Acetyltryptophan

31

Dihydrojasmonic acid

211.13

601.25

$-$

$344.07 \quad 625.18$

Cyclic GMP

33

12-Hydroxydodecanoic acid

215.1

34

NAGly

35

L-A-Lysophosphatidylserine

N-Arachidonoyl Dihydroxypropylamine

524.30

823.98

$376.29 \quad 825.78$

$\begin{array}{lllll}360.25 & 794.64 & - & \mathrm{C}_{22} \mathrm{H}_{35} \mathrm{NO}_{3}\end{array}$

$2.005 \quad 0.000$

1.6430 .003
$\mathrm{C}_{24} \mathrm{H}_{48} \mathrm{NO}_{9} \mathrm{P}$

$\mathrm{C}_{23} \mathrm{H}_{39} \mathrm{NO}_{3}$
$1.246 \quad 0.034$

$1.348 \quad 0.020$

$1.731 \quad 0.002$

$1.780 \quad 0.001$

$\uparrow \#$

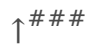

$\downarrow^{\#}$

\#\#

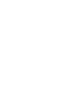

$\uparrow$ : the metabolite was up-regulated; $\downarrow$ : the metabolite was down-regulated.

${ }^{\#} p<0.05,{ }^{\# \#} p<0.01,{ }^{\# \# \#} p<0.001$, compared with control group.

${ }^{*} p<0.05,{ }^{* *} p<0.01,{ }^{* * *} p<0.001$, compared with model group.

Table 2 Dysregulated Proteins in different groups 


\begin{tabular}{|c|c|c|c|c|c|c|}
\hline No. & Accession & Gene Symbol & Description & $\begin{array}{c}\text { Control } \\
\text { vs } \\
\text { Model }\end{array}$ & $\begin{array}{l}\text { Model } \\
\text { vs FA }\end{array}$ & $\begin{array}{l}\text { Model } \\
\text { vs FB }\end{array}$ \\
\hline 1 & NP_001014348 & $\operatorname{srpx}$ & $\begin{array}{l}\text { sushi repeat-containing protein SRPX } \\
\text { precursor }\end{array}$ & $\downarrow^{\# \#}$ & $\uparrow^{* *}$ & $\uparrow^{*}$ \\
\hline 2 & NP_001028768 & mrps7 & $\begin{array}{c}28 \mathrm{~S} \text { ribosomal protein S7, mitochondrial } \\
\text { precursor }\end{array}$ & $\uparrow \# \#$ & $\downarrow^{* * *}$ & $\downarrow^{* * *}$ \\
\hline 3 & NP_001077285 & zgc:162509 & uncharacterized protein LOC553299 & $\uparrow \# \#$ & $\downarrow^{* *}$ & $\downarrow^{* *}$ \\
\hline 4 & NP_001268407 & $\operatorname{col} 2 \mathrm{a} 1 \mathrm{~b}$ & collagen, type II, alpha $1 \mathrm{~b}$ precursor & $\uparrow \# \#$ & $\downarrow^{* *}$ & $\downarrow^{* * *}$ \\
\hline 5 & NP_571742 & $\operatorname{gch} 2$ & GTP cyclohydrolase 1 & $\uparrow \# \#$ & $\downarrow^{* * *}$ & $\downarrow^{* *}$ \\
\hline 6 & NP_942574 & wdr3 & WD repeat-containing protein 3 & $\uparrow^{\# \#}$ & $\downarrow^{*}$ & $\downarrow^{*}$ \\
\hline 7 & NP_956140 & ptpn11a & $\begin{array}{c}\text { tyrosine-protein phosphatase non-receptor } \\
\text { type } 11\end{array}$ & $\uparrow^{\#}$ & $\downarrow^{*}$ & $\downarrow^{*}$ \\
\hline 8 & NP_956258 & rcvrn2 & recoverin 2 & $\downarrow^{\#}$ & $\uparrow^{* *}$ & $\uparrow^{* *}$ \\
\hline 9 & NP_991174 & nit2 & omega-amidase NIT2 & $\uparrow^{\#}$ & $\downarrow^{*}$ & $\downarrow^{*}$ \\
\hline 10 & NP_997744 & col9a2 & collagen alpha-2(IX) chain precursor & $\downarrow^{\# \#}$ & $\uparrow^{* * *}$ & $\uparrow^{* * *}$ \\
\hline 11 & NP_998429 & col9a1b & collagen type IX alpha I precursor & $\uparrow^{\# \#}$ & $\downarrow^{* *}$ & $\downarrow^{* * *}$ \\
\hline 12 & XP_021324964 & nme3 & nucleoside diphosphate kinase 3 isoform X1 & $\uparrow \# \# \#$ & $\downarrow^{* * *}$ & $\downarrow^{* * *}$ \\
\hline 13 & XP_695887 & scaf4a & splicing factor, arginine/serine-rich 15 & $\downarrow^{\#}$ & $\uparrow^{*}$ & $\uparrow^{* *}$ \\
\hline 14 & NP_001002461 & $\operatorname{txn}$ & thioredoxin & $\downarrow^{\# \#}$ & $\uparrow^{*}$ & - \\
\hline 15 & NP_001003625 & nup85 & nuclear pore complex protein Nup85 & $\uparrow^{\#}$ & $\downarrow^{* *}$ & - \\
\hline 16 & NP_001004660 & snrpg & small nuclear ribonucleoprotein G isoform 2 & $\downarrow^{\#}$ & $\uparrow^{*}$ & - \\
\hline 17 & NP_001006043 & ctsz & cathepsin Z precursor & $\downarrow^{\#}$ & $\uparrow^{*}$ & - \\
\hline 18 & NP_001017899 & capns1a & calpain small subunit 1 & $\downarrow^{\#}$ & $\uparrow^{* *}$ & - \\
\hline 19 & NP_001019906 & eif3m & $\begin{array}{c}\text { eukaryotic translation initiation factor } 3 \\
\text { subunit } \mathrm{M}\end{array}$ & $\downarrow^{\# \#}$ & $\uparrow^{* *}$ & - \\
\hline 20 & NP_001020680 & ppp6r2a & $\begin{array}{c}\text { serine/threonine-protein phosphatase } 6 \\
\text { regulatory subunit } 2\end{array}$ & $\uparrow^{\# \#}$ & $\downarrow^{* *}$ & - \\
\hline 21 & NP_001038800 & rpl2211 & $\begin{array}{l}\text { 60S ribosomal protein L22-like } 1 \\
\text { Page } 29 / 38\end{array}$ & $\downarrow^{\# \# \#}$ & $\uparrow^{* * *}$ & - \\
\hline
\end{tabular}




\begin{tabular}{|c|c|c|c|c|c|}
\hline 22 & NP_001071203 & bop1 & ribosome biogenesis protein bop1 & $\uparrow^{\#}$ & $\downarrow^{* *}$ \\
\hline 23 & NP_001082840 & mrps30 & $39 S$ ribosomal protein S30, mitochondrial & $\downarrow^{\#}$ & $\uparrow^{*}$ \\
\hline 24 & NP_001093210 & matn1 & cartilage matrix protein precursor & $\downarrow^{\#}$ & $\uparrow^{* * *}$ \\
\hline 25 & NP_001096604 & crygm $2 \mathrm{~d} 16$ & crystallin, gamma M2d16 & $\downarrow^{\# \#}$ & $\uparrow^{* * *}$ \\
\hline 26 & NP_001103591 & $\operatorname{rps} 23$ & 40S ribosomal protein S23 & $\downarrow^{\#}$ & $\uparrow^{*}$ \\
\hline 27 & NP_001166027 & bxdc2 & ribosome biogenesis protein BRX1 homolog & $\uparrow^{\# \#}$ & $\downarrow^{* *}$ \\
\hline 28 & NP_001289671 & atp5f1e & ATP synthase subunit epsilon, mitochondrial & $\downarrow^{\# \#}$ & $\uparrow^{* *}$ \\
\hline 29 & NP_001338629 & LOC100330864 & $\begin{array}{l}\text { ribonucleoside-diphosphate reductase subunit } \\
\text { M2 isoform } 1\end{array}$ & $\uparrow^{\#}$ & $\downarrow^{*}$ \\
\hline 30 & NP_571328 & opn1 mw1 & green-sensitive opsin-1 & $\downarrow^{\# \#}$ & $\uparrow^{*}$ \\
\hline 31 & NP_956159 & $\operatorname{cdc} 421$ & cell division control protein 42 homolog & $\downarrow^{\#}$ & $\uparrow^{*}$ \\
\hline 32 & NP_956500 & erap1b & $\begin{array}{c}\text { endoplasmic reticulum aminopeptidase } 1 \\
\text { precursor }\end{array}$ & $\downarrow^{\#}$ & $\uparrow^{*}$ \\
\hline 33 & NP_957036 & rps26l & ribosomal protein S26 & $\downarrow^{\#}$ & $\uparrow^{* *}$ \\
\hline 34 & NP_957153 & slc25a20 & $\begin{array}{c}\text { mitochondrial carnitine/acylcarnitine carrier } \\
\text { protein }\end{array}$ & $\downarrow^{\#}$ & $\uparrow^{* *}$ \\
\hline 35 & NP_958493 & $\mathrm{pfn} 2$ & profilin 2 & $\downarrow^{\# \#}$ & $\uparrow^{*}$ \\
\hline 36 & NP_958500 & $\operatorname{arpc1a}$ & actin-related protein $2 / 3$ complex subunit $1 \mathrm{~A}$ & $\downarrow^{\#}$ & $\uparrow^{* *}$ \\
\hline 37 & NP_963878 & $\operatorname{rpl12}$ & 60S ribosomal protein L12 & $\downarrow^{\# \#}$ & $\uparrow^{*}$ \\
\hline 38 & NP_991317 & psmd10 & $\begin{array}{l}\text { 26S proteasome non-ATPase regulatory } \\
\text { subunit } 10\end{array}$ & $\downarrow^{\#}$ & $\uparrow^{*}$ \\
\hline 39 & NP_997743 & mcmbp & $\begin{array}{c}\text { mini-chromosome maintenance complex- } \\
\text { binding protein }\end{array}$ & $\downarrow^{\# \#}$ & $\uparrow^{*}$ \\
\hline 40 & NP_997750 & ist1 & IST1 homolog isoform 1 & $\downarrow^{\# \#}$ & $\uparrow^{* * *}$ \\
\hline 41 & NP_998569 & $\operatorname{lipf}$ & $\begin{array}{c}\text { lysosomal acid lipase/cholesteryl ester } \\
\text { hydrolase precursor }\end{array}$ & $\downarrow^{\#}$ & $\uparrow^{*}$ \\
\hline 2 & NP_998605 & plrg1 & pleiotropic regulator 1 & $\uparrow \# \#$ & $\downarrow^{* *}$ \\
\hline
\end{tabular}




\begin{tabular}{|c|c|c|c|c|c|c|}
\hline 43 & NP_999858 & $\operatorname{lgals} 3 b$ & galectin-3 & $\downarrow^{\# \#}$ & $\uparrow^{* * *}$ & - \\
\hline 44 & NP_999977 & epb4113b & $\begin{array}{l}\text { erythrocyte membrane protein band 4.1-like } \\
3 \mathrm{~b}\end{array}$ & $\uparrow^{\#}$ & $\downarrow^{*}$ & - \\
\hline 45 & XP_001923961 & nxf1 & nuclear RNA export factor 1 & $\uparrow^{\# \#}$ & $\downarrow^{*}$ & - \\
\hline 46 & XP_005159500 & $\operatorname{col} 11 \mathrm{a} 2$ & collagen alpha-2 (XI) chain isoform X1 & $\downarrow^{\# \#}$ & $\uparrow^{*}$ & - \\
\hline 47 & XP_005163206 & $\operatorname{tnni2a.4}$ & troponin I, skeletal, fast $2 \mathrm{a} .4$ isoform X1 & $\uparrow \# \#$ & $\downarrow^{* * *}$ & - \\
\hline 48 & XP_017208249 & coro6 & coronin-6 isoform X1 & $\downarrow^{\#}$ & $\uparrow^{* * *}$ & - \\
\hline 49 & XP_021322373 & arhgef1b & $\begin{array}{c}\text { rho guanine nucleotide exchange factor } 1 \\
\text { isoform X1 }\end{array}$ & $\downarrow^{\# \#}$ & $\uparrow^{* *}$ & - \\
\hline 50 & XP_685270 & sgca & alpha-sarcoglycan & $\uparrow^{\#}$ & $\downarrow^{* *}$ & - \\
\hline 51 & XP_691943 & si:dkey-23a13.8 & histone H2B 1/2-like & $\uparrow \# \#$ & $\downarrow^{* *}$ & - \\
\hline 52 & NP_001019612 & psma2 & proteasome alpha 2 subunit & $\uparrow^{\#}$ & - & $\downarrow^{*}$ \\
\hline 53 & NP_001071216 & $\operatorname{scg} 2 b$ & secretogranin-2 precursor & $\downarrow^{\# \#}$ & - & $\uparrow^{* *}$ \\
\hline 54 & NP_001313480 & $\begin{array}{l}\text { si:dkey- } \\
251 \mathrm{i} 10.2\end{array}$ & si:dkey-251i10.2 precursor & $\downarrow^{\#}$ & - & $\uparrow^{*}$ \\
\hline 55 & NP_957419 & spag 7 & sperm-associated antigen 7 homolog & $\uparrow^{\#}$ & - & $\downarrow^{*}$ \\
\hline 56 & XP_005155974 & $\begin{array}{l}\text { si:ch211- } \\
222121.1\end{array}$ & prothymosin alpha & $\uparrow$ १\#\# & - & $\downarrow^{* *}$ \\
\hline
\end{tabular}

$\uparrow$ : the protein was up-regulated; $\downarrow$ : the protein was down-regulated.

${ }^{\#} p<0.05,{ }^{\# \#} p<0.01,{ }^{\# \# \#} p<0.001$, compared with control group.

${ }^{*} p<0.05,{ }^{* *} p<0.01,{ }^{* * *} p<0.001$, compared with model group.

\section{Figures}




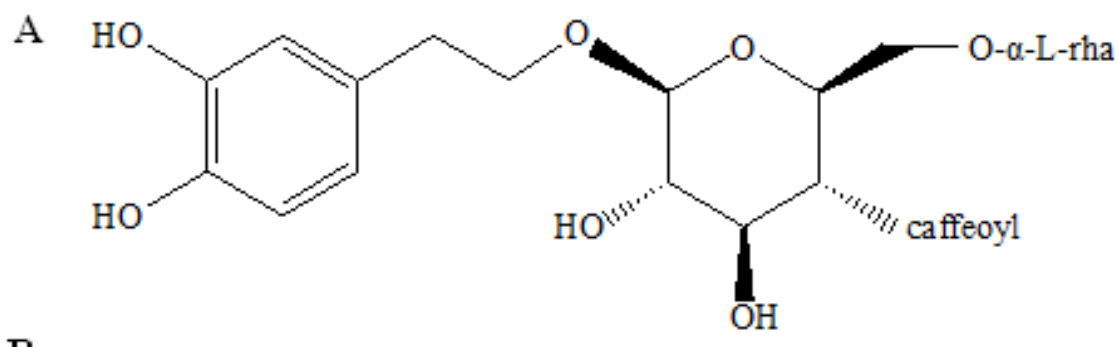

B<smiles>[CH]OC[C@H]1O[C@@H](OCCc2ccc(O)c(O)c2)[C@H](O)[C@@H](O)[C@@H]1CC(=O)O</smiles>

\section{Figure 1}

Chemical structures of Forsythiaside $A(A)$ and Forsythiaside $B(B)$.

A

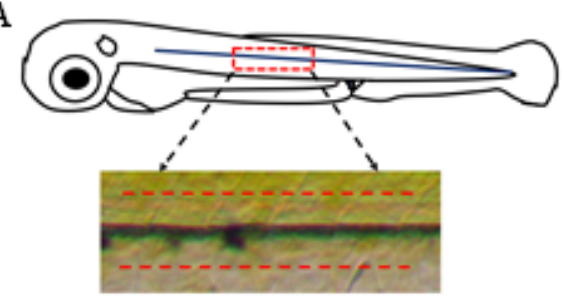

C

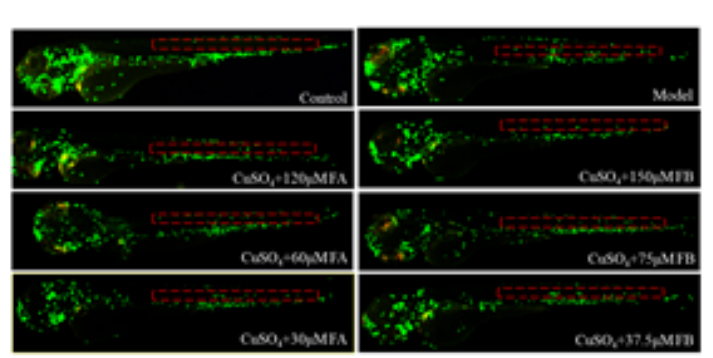

B
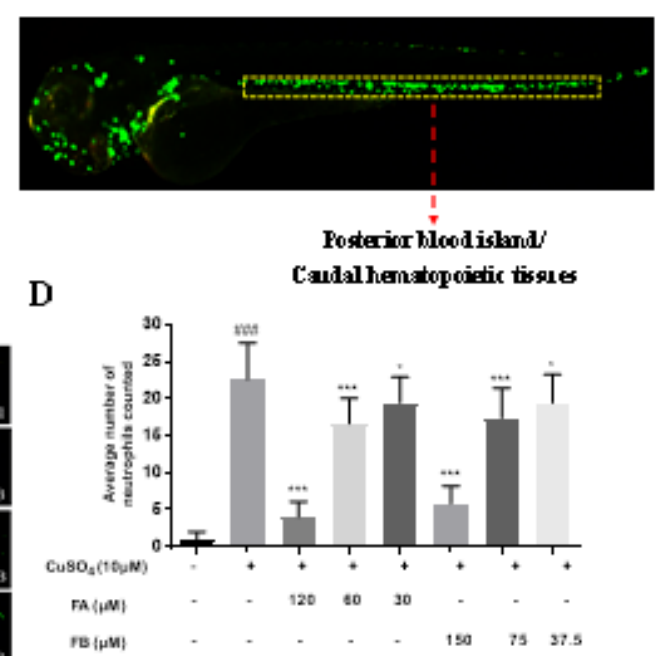

\section{Figure 2}

FA and FB inhibit neutrophils recruitment to the injury site in CuSO4 treated zebrafish. (A) A general view of 3 dpf zebrafish larvae. The boxed area referred to the horizontal lateral line, and neutrophils within the horizontal line (dotted red lines) were calculated in quantification experiments. (B) The area of the posterior blood island or caudal hematopoietic tissues (the boxed region with dotted yellow lines) in untreated Transgenic (mpx:EGFP) zebrafish. Most leukocytes in this development stage were distributed in this area. (C) Microphotographs showing neutrophils migration in control, model, FA and FB group in different 
concentrations (neutrophils of $3 \mathrm{dpf}$ transgenic (mpx:EGFP) zebrafish exhibiting green fluorescence). The red dotted lines showed the location of the horizontal muscles of zebrafish. (D) FA and FB dose-dependently reduced the number of neutrophils counted in the injury site. $\# p<0.05$, \#\#p $<0.01$, \#\#\#p<0.001, compared with control group; $* p<0.05, * \star p<0.01, * \star \star p<0.001$, compared with model group. Data were shown as mean \pm S.D, $n=35$.
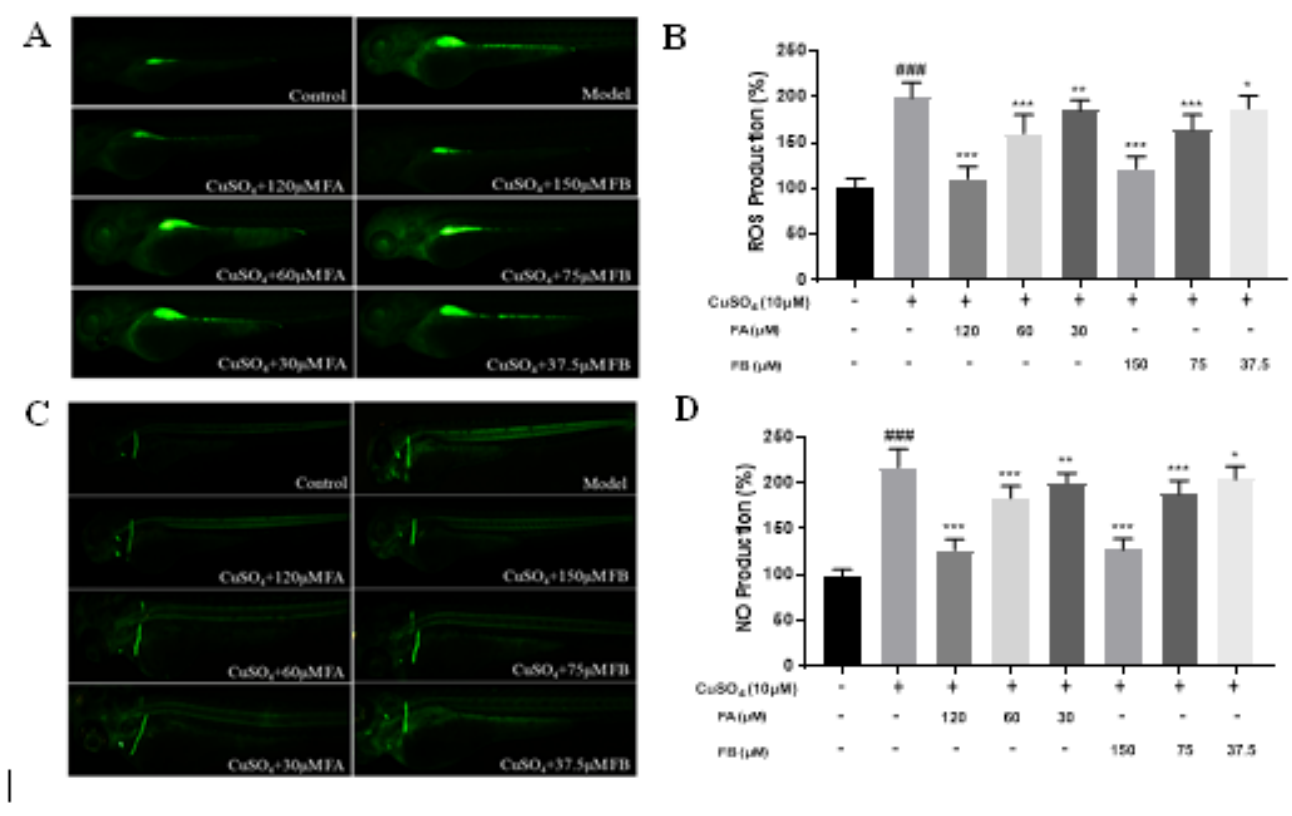

\section{Figure 3}

FA and FB alleviated inflammation in zebrafish by inhibiting CuSO4-induced ROS and NO generation. (A) Microphotographs documenting ROS production in control, model, FA and FB group in different concentrations ( 3 dpf wild-type AB strain zebrafish larvae). (B) FA and FB dose-dependently reduced CuSO4induced ROS generation. (C) Microphotographs documenting production of $\mathrm{NO}$ in control, model, FA and FB group in different concentrations ( 3 dpf wild-type AB strain zebrafish larvae). (D) FA and FB dose-dependently reduced CuSO4-induced NO generation. \#p < 0.05, \#\#p < 0.01, \#\#\#p<0.001, compared with control group; *p $<0.05,{ }^{*} \mathrm{p}<0.01,{ }^{\star} * \star \mathrm{p}<0.001$, compared with model group. Data were shown as mean $\pm S . D, n=35$. 

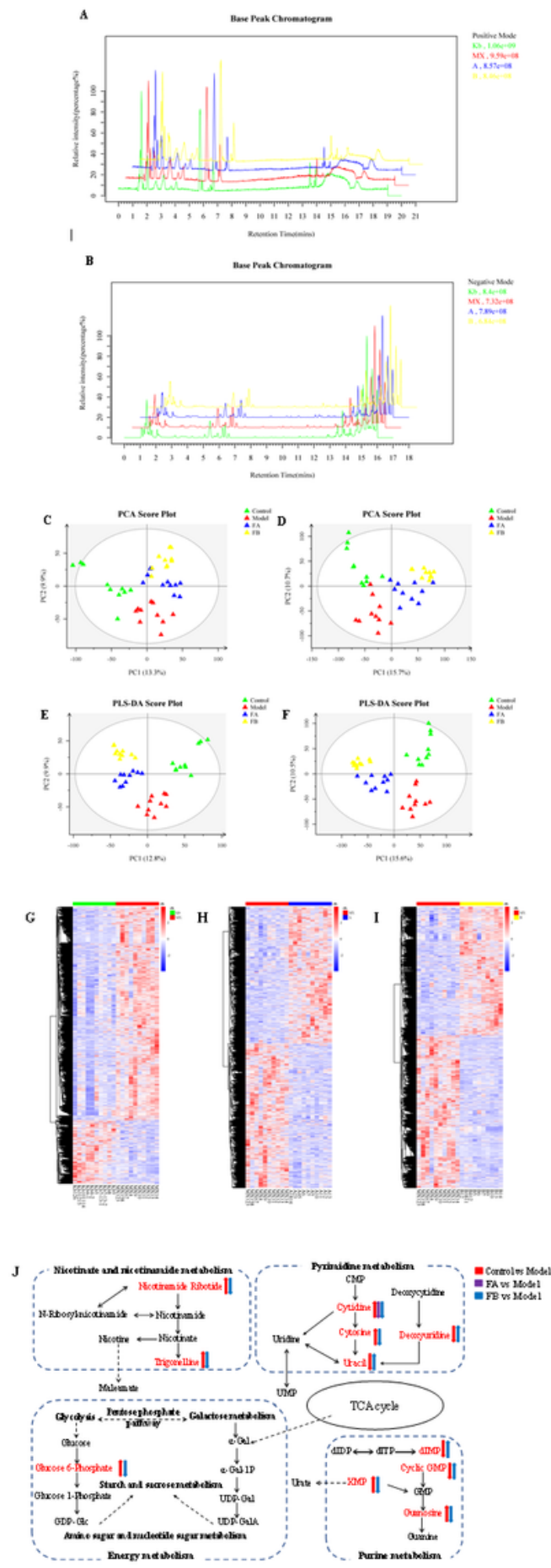

\section{Figure 4}

Metabolomic analyses of zebrafish larvae. Representative Base Peak Chromatogram (BPC) of zebrafish in control, model, FA and FB group obtained from positive ion mode (A) and negative ion mode (B). PCA score plot of zebrafish metabolites from positive ion mode (C) and negative ion mode (D). PLS-DA score plot of zebrafish metabolites from positive ion mode $(E)$ and negative ion mode $(F)$. Heat-map of metabolites of control vs. model $(\mathrm{G})$, model vs. FA (H), model vs. FB (I). Rows: differential metabolites; Columns: zebrafish samples. The rectangle in different color represented the expression level of metabolites. Highest in red; 
lowest in blue; mean in white. $(\mathrm{J})$ Metabolic pathways (bold) participating in the anti-inflammatory action of FA and FB in CuSO4-induced inflammation in zebrafish. The metabolites (red) were identified biomarkers in the present study. Arrows nearby metabolites indicated the relative expressions of metabolites of control vs. model, model vs. FA, model vs. FB.
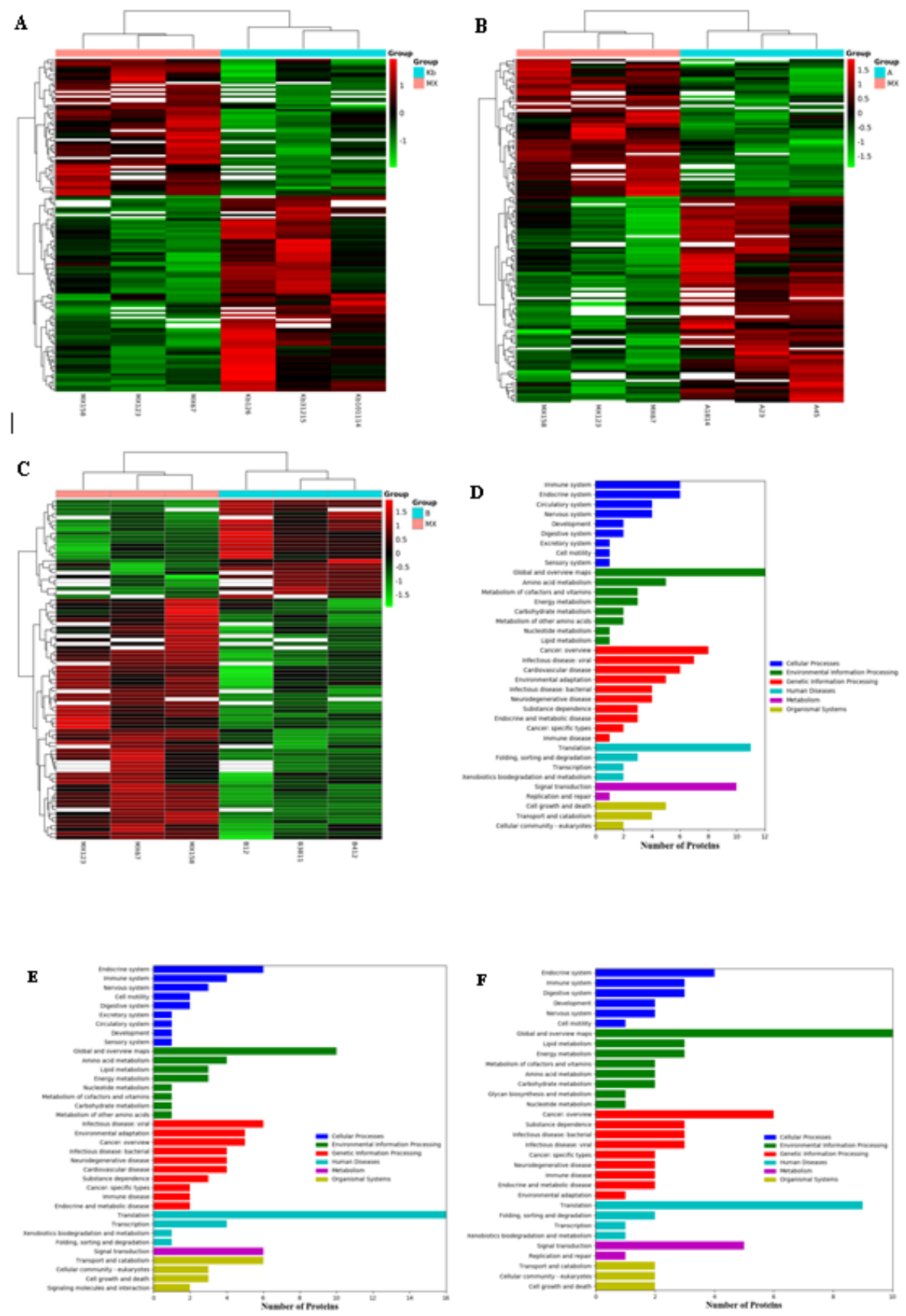

\section{Figure 5}

Proteomic analyses of zebrafish larvae. Heat-map of differential proteins of control vs. model (A), model vs. FA (B), model vs. FB (C). Rows: differential proteins; Columns: zebrafish samples. The rectangle in different 
color represented the expression level of proteins. Highest in red; lowest in green; mean in white. The KEGG pathway classification of differential proteins of control vs. model (D), model vs. FA (E), model vs. FB (F). Rows: number of proteins; Columns: KEGG pathway classification.
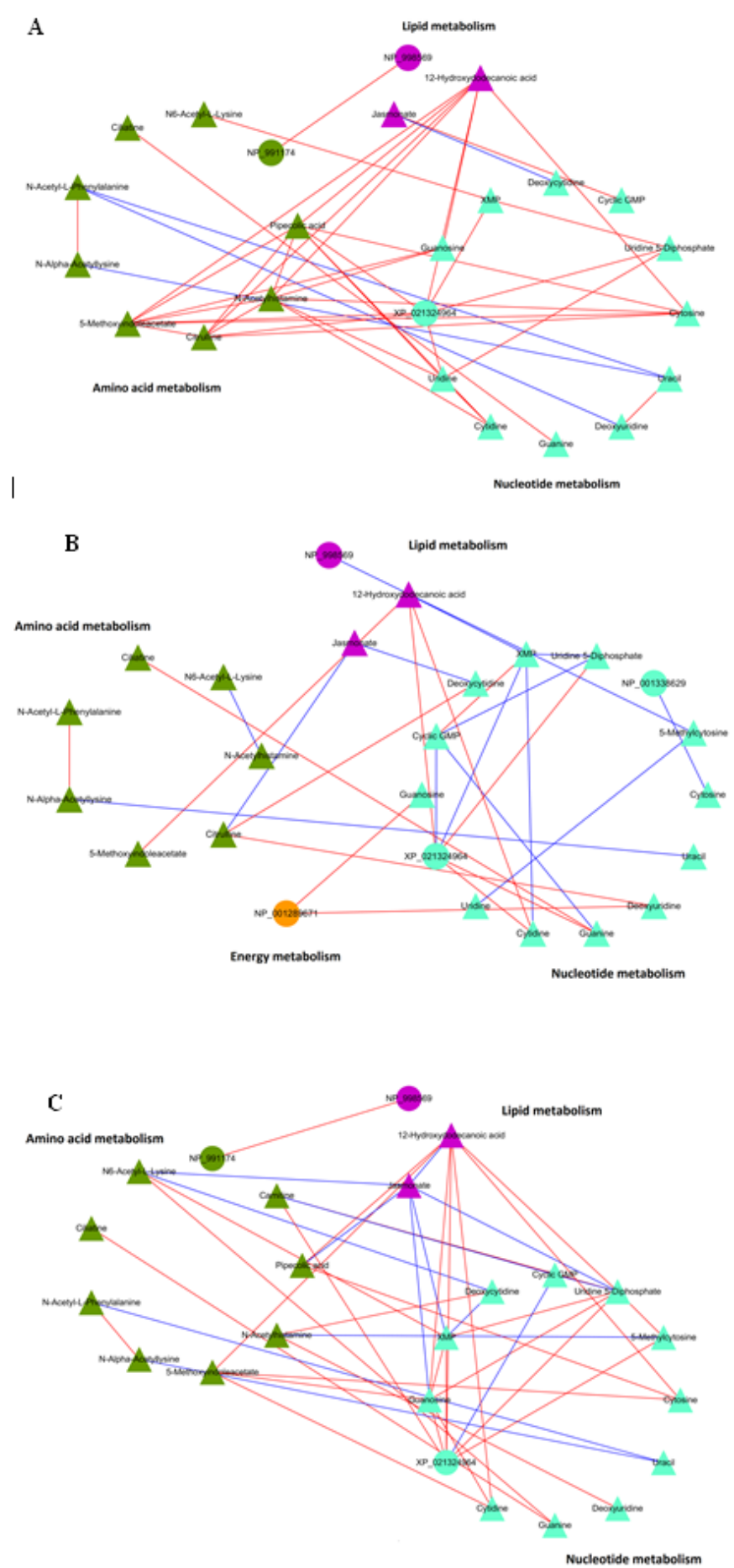

\section{Figure 6}

Interactive network construction by integrated metabolomics and proteomics analyses. Interactive network of metabolites and proteins of model group compared with control (A), FA (B), and FB group (C). Triangles and circles in different colors represented metabolites and proteins in different metabolic pathways, respectively. 


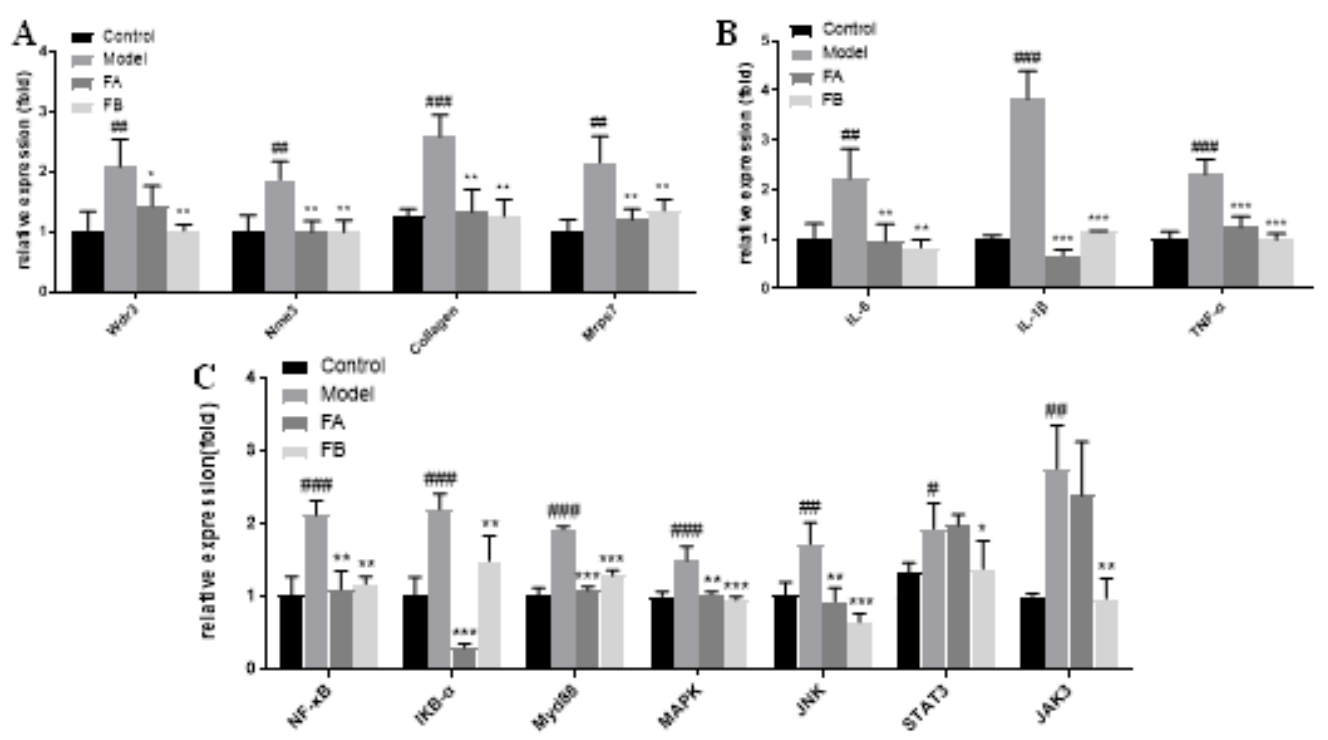

\section{Figure 7}

Relative expressions of mRNA in different groups detected by qRT-PCR. (A) The mRNA expressions of Wdr3, Nme3, Collagen, Mrps7 of control, model, FA and FB group. (B) The mRNA expressions of IL-6, IL-1 $\beta$, TNF-a of control, model, FA and FB group. (C) The mRNA expressions of genes involved in NF-KB, MAPK, JAK-STAT signaling pathways of control, model, FA and FB group. \#p $<0.05$, \#\#p $<0.01$, \#\#\#p $<0.001$, compared with control group; ${ }^{\star} p<0.05,{ }^{\star *} p<0.01,{ }^{\star \star \star} p<0.001$, compared with model group. Data were shown as mean \pm S.D, $\mathrm{n}=35$.

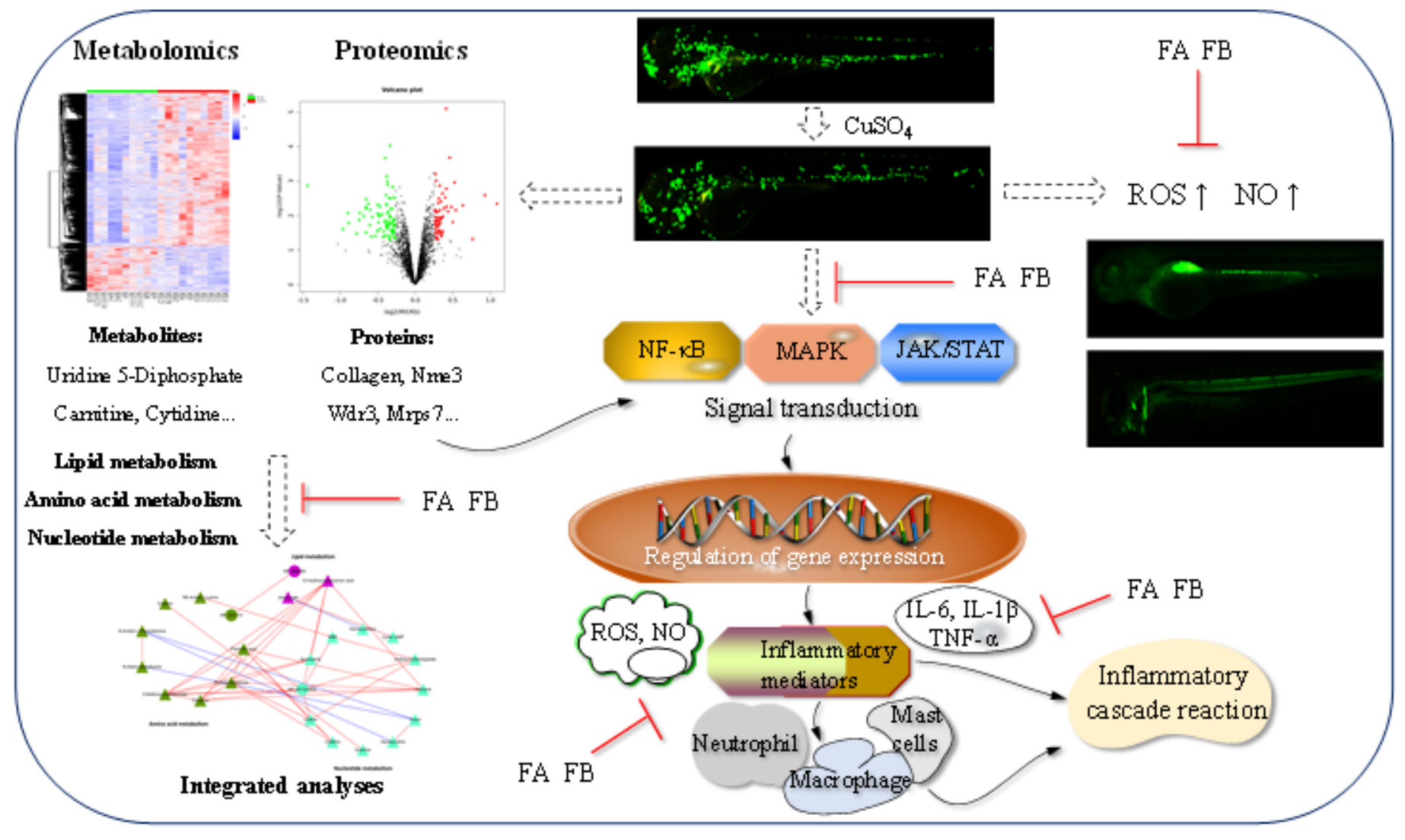


Figure 8

A probable mechanism of FA and FB in the protection of CuSO4-induced inflammation in zebrafish. FA and FB administration resulted in reductions of ROS and NO, alterations of various metabolites, proteins and gene products induced by CuSO4, thus reducing inflammation. This diagram showed the multi-component and multi-regulatory therapeutic mechanism of FA and FB against CuSO4-induced inflammation in zebrafish.

\section{Supplementary Files}

This is a list of supplementary files associated with this preprint. Click to download.

- Additionalfile2.docx

- Additionalfile4.docx

- Additionalfile3.docx

- Additionalfile5.docx

- Additionalfile1.docx 